\title{
Zebrafish grainyhead-like1 is a common marker of different non-keratinocyte epidermal cell lineages, which segregate from each other in a Foxi3-dependent manner
}

\author{
MARTINA JÄNICKE ${ }^{1, \#, ~ B J O ̈ R N ~ R E N I S C H ~}{ }^{1,2,3, \#}$ and MATTHIAS HAMMERSCHMIDT*,1,2,3,4 \\ ${ }^{1}$ Max-Planck-Institute of Immunobiology, Freiburg, ${ }^{2}$ Institute for Developmental Biology, \\ ${ }^{3}$ Cologne Excellence Cluster on Cellular Stress Responses in Aging-Associated Diseases and \\ ${ }^{4}$ Center for Molecular Medicine Cologne, University of Cologne, Cologne, Germany
}

\begin{abstract}
Grainyhead/CP2 transcription factor family members are widely conserved among the animal kingdom and have been implicated in different developmental processes. Thus far, nothing has been known about their roles in zebrafish. Here we identify seven zebrafish grainyhead-like (grhl) / cp2 genes, with focus on grh/1, which is expressed in the periderm and in epidermal ionocyte progenitors, but downregulated when ionocytes differentiate. In addition, expression was detected in other "non-keratinocyte" cell types of the epidermis, such as pvalb8expressing cells, which according to our lineage tracing experiments are derived from the same pool of progenitor cells like keratinocytes and ionocytes. Antisense morpholino oligonucleotidebased loss-of-function analysis revealed that grhl1 is dispensable for the development and function of all investigated epidermal cell types, but required as a negative regulator of its own transcription during ionocyte differentiation. Knockdown of the transcription factor Foxi3a, which is expressed in a subset of the grh/1 population, caused a loss of ionocytes and a corresponding increase in the number of pvalb8-expressing cells, while leaving the number of grhl1-positive cells unaltered. We propose that grh/1 is a novel common marker of all or most "non-keratinocyte" epidermal progenitors, and that the sub-functionalisation of these cells is regulated by differential positive and negative effects of Foxi3 factors.
\end{abstract}

KEY WORDS: grainyhead, skin, ionocyte, foxi3, p63, pvalb8, zebrafish

\section{Introduction}

During embryonic and larval development, the zebrafish epidermis consists of two cell layers, the basal layer, which originates from the ventral/non-neural ectoderm of the gastrula embryo, and the superficial layer, the periderm, which is derived from the embryonic enveloping layer (EVL), a lineage that segregates from the deep cells (including the epidermal ectoderm) during pregastrula stages (Kimmel et al., 1990; Le Guellec et al., 2004; Sonawane et al., 2005). Both layers predominantly consist of keratin-synthesising epithelial cells. In addition to these keratinocytes, the epidermis of teleosts contains a range of different cell types, such as ionocytes, which perform active ion transport and are involved in osmoregulation, mucus-secreting goblet cells, and other secretory cell types such as sacciform cells and club cells (for review, see Whitear, 1986). In zebrafish embryos and larvae, epidermal ionocytes are distributed in a punctate pattern throughout the epidermis of the trunk and tail. They are comprised of at least two types, $\mathrm{Na}^{+}, \mathrm{K}^{+}$-ATPase-rich $(\mathrm{NaR})$ cells and $\mathrm{H}^{+}-\mathrm{ATPase}$-rich $(\mathrm{HR})$ cells, which are characterised by the expression of the specific ATPase genes atp $1 b 1 \mathrm{~b}$ and

\footnotetext{
Abbreviations used in this paper: BrdU, bromodeoxyuridine; dpf, days post fertilization; EVL, enveloping layer; GFP, green fluorescent protein; grhl, grainyhead-like; hpf, hours post fertilization; HR, $\mathrm{H}^{+}$-ATPase-rich; MO, morpholino oligonucleotide; $\mathrm{NaR}, \mathrm{Na}^{+}, \mathrm{K}^{+}$-ATPase-rich; $\mathrm{NCC}, \mathrm{Na}^{+} / \mathrm{Cl}^{-}$ cotransporter expressing; pNB, postembryonic neuroblasts; RT-PCR, reverse transcription polymerase chain reaction.
}

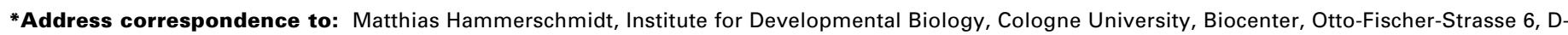

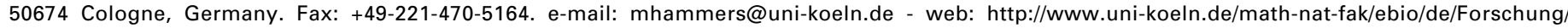
Hammerschmidt/hammerschmidt.html - \#Note: Both authors contributed equally to the work
}

Supplementary Material (8 figures + 2 tables) for this paper is available at: http://dx.doi.org/10.1387/ijdb.092877mj 
atp6v1al, respectively (Lin et al., 2006; reviewed in Hwang and Lee, 2007). In addition, epithelial mucous cells have been described (Hsiao et al., 2007; Shih et al., 2007). The cell bodies of these different "non-keratinocyte" cells are located within the basal layer of the epidermis, with characteristic protrusions that extend through the outer periderm towards the surface of the fish (Whitear, 1986).

Cell lineage tracing experiments have shown that basal keratinocytes and ionocytes are derived from a common pool of precursor cells from the ventral ectoderm (Jänicke et al., 2007). Foxi3 transcription factors are necessary for ionocyte specification, while Notch ligands specifically made in developing ionocytes prevent a further expansion of the ionocyte lineage by signalling to neighbouring epidermal cells, thereby preventing foxi3expression in these cells and allowing them to adopt a keratinocyte fate (lateral inhibition) (Jänicke et al., 2007; Hsiao et al., 2007). Within the keratinocyte lineage, the transcription factor DNp63 is crucial for proliferation, although most likely dispensable for differentiation of these cells (Bakkers et al., 2002; Lee and Kimelman, 2002). However, the exact mechanisms controlling the initial segregation steps of the different epidermal cell lineages are still poorly understood.

Members of the Grainyhead/CP2 transcription factor family are highly conserved across the animal kingdom and are involved in a variety of developmental processes. The family is split into two divisions, the Grainyhead and the CP2 subgroup. The first Grainyhead protein was identified in Drosophila melanogaster, based on its binding to neuronal cis elements, and initially called NTF-1 or Elf-1, but renamed Grainyhead according to its mutant (Dynlacht et al., 1989; Bray et al., 1989; Bray and Kafatos, 1991), while CP2 was first discovered in mouse, based on its binding to the globin promoter (Lim et al., 1992).

The fruitfly has one member of each subgroup, D-grainyhead and $D$-Cp2. In mammals, three grainyhead-like genes have been described, Grainyhead-like 1 (also known as Mother-of-grainyhead or Lbp-32), Grainyhead-like2(also known as Brother-of-mammalian-grainyhead, or Cp2-like3) and grainyhead-like3 (also known as Sister-of-mammalian-grainyhead, Cp2-like4 or Get-1). The CP2 subgroup also consists of three genes in mammals, Cp2, Cp2-like 1and Lbp-1a (Venkatesan etal., 2003; Wilanowski etal., 2002).

In Drosophila, tissue-specific splice variants of grainyheadare required for epidermal specification (Bray and Kafatos, 1991) as well as neuroblast differentiation (Maurange et al., 2008; Almeida and Bray, 2005; Cenci and Gould, 2005). Mammalian Grainyheadlike 2 is required for maturation of fluid/solute transporting epithelial ducts in both the salivary gland and the kidney in mice (Yamaguchi et al., 2006) and has also been linked to age-related deafness in humans (Van Laer et al., 2008). Mice deficient in Grainyhead-like3 show abnormalities in the granular and cornified layer of the skin and in cutaneous wound healing, indicating that the gene is required for proper terminal differentiation of the epidermis and for epithelial morphogenesis (Ting et al., 2005; Hislop et al., 2006; Yu et al., 2006; Yu et al., 2008). A similar differentiation-promoting role has been demonstrated for Xenopus Grhl2, which upon forced expression induces genes specific for differentiated superficial cells in the deep proliferating epidermal cells (Chalmers et al., 2006). In addition, Xenopus Grh/1 disruption leads to defects in epidermal differentiation, as indi- cated by the loss of keratin genes expression (Tao et al., 2005), while mouse Grh/1 mutants display skin and hair defects partly caused by reduced expression of the genes encoding desmosomal cadherins (Wilanowski et al., 2008).

Here we report the identification of seven members of the Grainyhead/CP2 family in the zebrafish genome. The genes cluster into the six already described subgroups, with grh/2as the only duplicated pair. The aim of this work was the analysis of grainyhead-like1 ( $g r h / 1)$, which is expressed in the periderm (outer layer of the embryonic skin) until 2 days post fertilisation (dpf), and in single cells spread throughout the epidermis of the developing embryo. Performing fluorescent double in situ hybridisations, we show that this punctate pattern reflects transient grh/1 expression in ionocyte progenitors, while expression is downregulated when ionocytes start to differentiate. In addition, grh/1 expression was detected in other non-keratinocyte skin cell types deriving from the same pool of epidermal progenitors, such as pvalb8expressing putative mucous cells. We propose that $g r h / 1$ marks the progenitor stages of various cell lineages in the skin of the developing zebrafish larvae. Furthermore, we show that loss of foxiza, which has a negative effect on ionocyte markers such as atp $1 b 1 b$ and atp6v1al, has a positive effect on pvalb8 expression, suggesting that Foxi3 transcription factors induce ionocyte differentiation in epidermal cells that would otherwise become pvalb8-expressing cells.

\section{Results}

\section{grainyhead-like1 (grhl1) is expressed in skin cells of the zebrafish larvae}

Performing in situhybridisations we found that during the first 3 days of development, $g r h / 1$ was expressed in a punctate pattern throughout the basal epidermis of the zebrafish larvae. During cleavage (Fig. 1A), blastula and gastrula stages (data not shown), no grh/1 expression was detectable, indicating the lack of maternal mRNA contribution. First specific expression was observed around the 2-somites stage in distinct cells spread across the yolk of the embryo (Fig. 1B), resembling the previously described pattern of the foxi3genes at the same stage (Solomon et al., 2003; Hsiao et al., 2007; Jänicke et al., 2007). As somitogenesis progresses, grh/1-positive cells also appeared in the basal epidermis over the developing yolk sac extension and trunk (Fig. 1 C,D) as well as in the olfactory placodes (Fig. 1C, arrowhead). In addition, very faint expression was detected throughout the entire skin of the larvae until $2 \mathrm{dpf}$. This staining was restricted to cells of the superficial layer (also called enveloping layer (EVL) or periderm), which are characterised by their hexagonal cell shape (Fig. $1 \mathrm{E}-\mathrm{E}^{\prime \prime}$, black arrows; compare with single basal cell showing strong grh/1 expression indicated with red arrow). At $3 \mathrm{dpf}$, the punctate expression of $g r h / 1$ over the trunk began to vanish, while expression started in the branchial arch regions (Fig. $1 \mathrm{~F}$ and $\mathrm{G}$ ). At $5 \mathrm{dpf}$, grh/1 was strongly expressed in the branchial arches as well as in the nasal pits (Fig. $1 \mathrm{H}$ and I). At all stages investigated, no signal was detected with a grh/1 sense probe (Fig. 1E' and data not shown).

\section{Seven members of the Grainyhead/Cp2-family can be identi- fied within the zebrafish genome}

To identify other zebrafish $\mathrm{grh} /$ genes, zebrafish genome 
databases were searched using BlastN and TBlastN with Drosophila grhand Drosophila cp2as queries. Seven genes located on five different chromosomes were identified (Fig. 1J). When a phylogenetic tree was constructed, including previously published sequences of Grhl/Cp2 proteins from human, mouse, chicken and Xenopus, the identified zebrafish genes clustered into the six already described subgroups of the vertebrate Grainyhead/CP2 family (Fig. 1J, Wilanowski et al., 2002; Venkatesan et al., 2003). For grh/1, which is located on chromosome 17, no paralogue was identified. In addition, only one zebrafish orthologue of each of mammalian Grainyhead-like3, Cp2, Cp2-like1 and Lbp-1a was identified on chromosome 17 (z-grh/3), chromosome 23 ( $z$ cp2), chromosome 9 (z-cp2-like 1) and chromosome $19(z-/ b p-1 a)$, respectively. However, two sequences, placed on chromosome 16 and 19, clustered with Grainyhead-like 2 proteins. These genes were therefore called $z$ grh/2a and $z$-grh/2b, respectively.

Transcripts of all genes except $z-/ b p-1 a$ could be detected by RT-PCR during the first three days of development (data not shown), but of the five other $\mathrm{grh} / \mathrm{cp} 2$ genes that were positive in the RT-PCR, only expression of $g r h / 2 a, g r h / 2 b$ and $g r h / 3$ was high enough for detection in our whole mount in situ hybridisations (Fig. $1 \mathrm{~K}-\mathrm{T}$ ). This difference in sensitivity of both methods has also been described by others (Kochilas et al., 2003; Zhao et al., 2005; Xu et al., 2006). While grh/2a expression was restricted to the olfactory and otic placodes as well as to the pronephros from mid somitogenesis until $1 \mathrm{dpf}$ (Fig. $1 \mathrm{~K}-\mathrm{L}$ ), grh/2bwas ubiquitously expressed during gastrulation and the first day of development (Fig. $1 \mathrm{M}-\mathrm{O}^{\prime}$ ). grh/3displayed expression in the periderm of the skin, which started at late gastrula stages (Fig. 1 P-Q') and persisted, although at lower levels, during segmentation (Fig. $1 \mathrm{~S}, \mathrm{~T}$ ), when the periderm showed strong grh/1 expression. In addition, but unlike $g r h / 1, g r h / 3$ was expressed in pharyngeal tissue of segmentation embryos (Fig. $1 \mathrm{R}$, arrow). In reverse, grh/3lacked the punctate expression in the basal epidermis displayed by grh/1(Fig. 1 S,T; data not shown). In the Zebrafish Model Organism Database ZFIN, the same expression pattern has been published for grh/3under the name si:dkey-221/4.7 (Thisse et al., 2001; http://zfin.org).

\section{grhl1 is expressed in ionocyte precursor cells but switched off in differentiated ionocytes}

The expression of grh/1 in single cells throughout the skin during the first three days of development, followed by appearance in

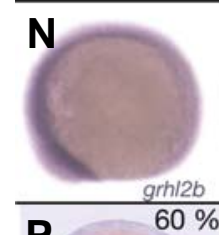

the branchial arch regions, resembles the expression pattern described for markers of differentiating HR- and NaR-type ionocytes (Varsamos et al., 2005; Hsiao et al., 2007; Jänicke et al., 2007).

We have previously shown that ionocytes are derived from the
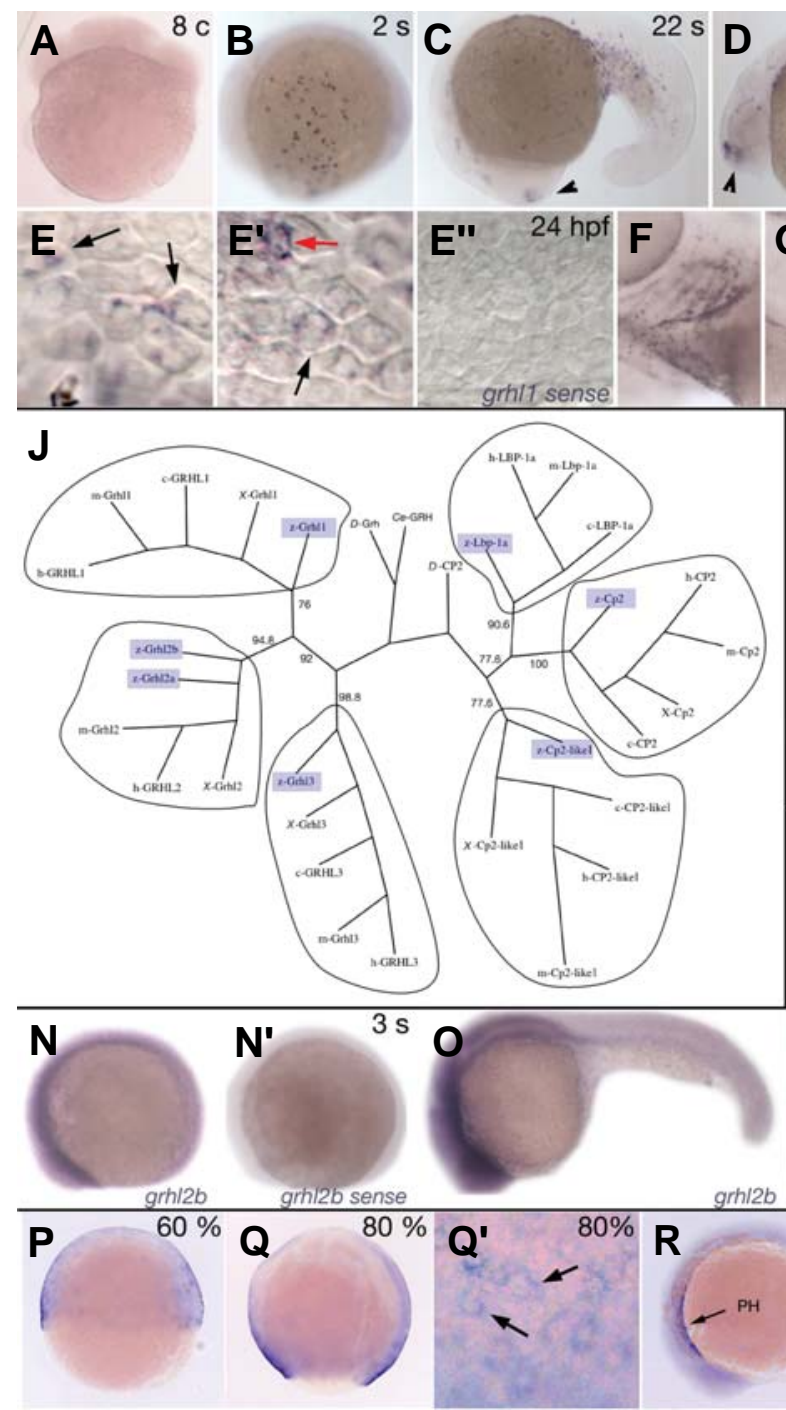

Fig.1. Of all zebrafish grhl-cp2 homologs, only grh/1 and grh/3 display specific expression in the embryonic skin. All panels except (J) show whole mount in situ hybridisations of wildtype embryos at stages indicated in the upper right corner and probes indicated in the lower right corner (c, cells; s, somites; \%, \% epiboly). (A-I) Expression of grhl1; (E') control staining with sense probe. Black arrowheads in $(C, D, H, L)$ point to the olfactory placodes. (E-E') High magnifications of the tail fin, with peridermal cells in focus (recognisable by their hexagonal shape). Black arrows point to staining in the superficial peridermal cells, the red arrow in (E') to a single cell in the underlying basal epidermis that expresses high levels of grhl1. (K-L) Staining for grhl2a. (M-O') Expression of grhl2b with an antisense (M-O) and sense probe (M'-O'). (P-T) Expression of grhl3, with $\left(Q^{\prime}\right)$ displaying a magnified view on the enveloping layer (future periderm; black arrows point to single cells) of the embryo shown in (Q). All panels except $\left(F, H_{,} K^{\prime}\right.$ ,T) show lateral views with anterior/vegetal to the left. $(\mathbf{F}, \mathbf{H})$ Ventral views with anterior to the left; $\left(K^{\prime}\right)$ is a posterior view with dorsal up, and (T) shows a transverse section at the trunk level. (J) An unrooted phylogenetic tree based on positional variation within regions of sequences that aligned across all identified (boxed) and published Grainyhead/CP2 proteins using the neighborjoining algorithm. Bootstrap values in \% based on 1000 replicates are indicated for the major branching points. Abbreviations: $O P$, otic placode; $P H$, pharynx; $P N$, pronephros. 

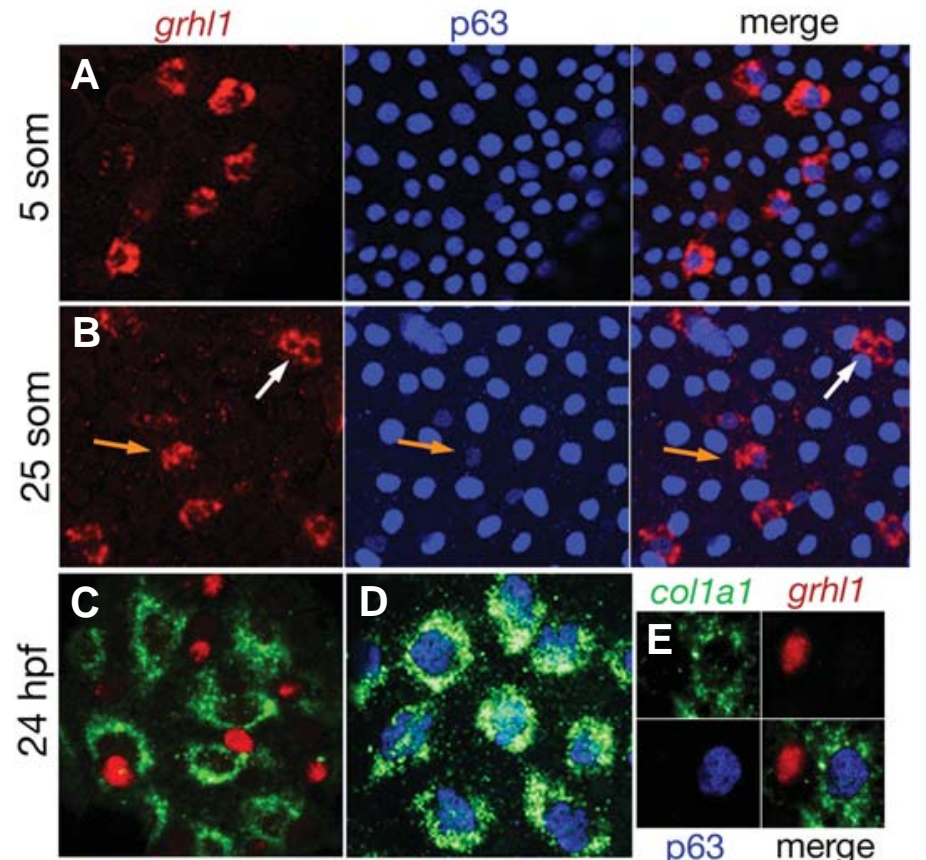

col1a1 + grhl1

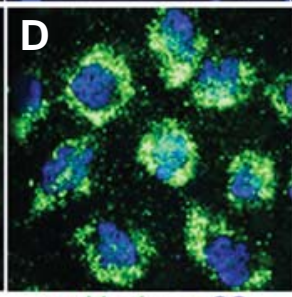

col1a1 + p63

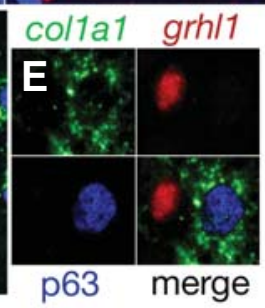

Fig. 2 (Above). grh/1 is transiently expressed in a subset of epidermal precursors, but absent from differentiated basal keratinocytes. (A,B) Fluorescent in situ hybridisation for grhl1 (in red) combined with immunostaining for p63 (in blue). (C) Double fluorescent in situ hybridization for col1a1 (in green) and grhl1 (in red). (D) Fluorescent in situ hybridisation for col1a1 (in green) combined with immunostaining for p63 (in blue). (E) A triple staining for col1a1 mRNA, grhl1 mRNA and p63 protein (three single channels plus merged image). For panels (C-E), embryos injected with grhl1 splice MO were used, leading to accumulation of grhl1 mRNA in the nucleus and facilitating the analysis of the obtained patterns (compare with Figs. 6 and S7).

Fig. 3 (Right). grhl1 is transiently expressed in ionocytes. All panels show double fluorescent in situ hybridisations with probes indicated above the panels. The right picture of each panel shows the merge of the two left pictures. Stages are indicated at the left. All panels show cells of wild-type embryos over the yolk sac (5-16 som) or over the anterior half of the trunk (22 som - 24 hpf). White arrows mark cells expressing only grhl1, green arrows mark cells only expressing foxi3a (B), foxi3b (D), or atp1b1b (F), and orange arrows mark cells co-expressing the two indicated genes.

same precursor cells as keratinocytes within the ventral ectoderm of the zebrafish gastrula (Jänicke et al., 2007). The transcription factor DNp63, a crucial regulator of keratinocyte proliferation, (Bakkers et al., 2002; Lee and Kimelman, 2002), is temporarily expressed in all epidermal progenitor cells of the non-neural ectoderm, while later, expression is only maintained in basal keratinocytes (Fig. 2D). Consistently, it is temporarily made in ionocyte progenitors, but lost after the onset of expression of foxi3a and foxi3b, essential regulators of $\mathrm{NaR}$ and $\mathrm{HR}$ ionocyte specification (Hsiao et al., 2007; Jänicke et al., 2007).

Fluorescent in situhybridisations for grh/1 in combination with anti-p63 immunostainings revealed that at early somite stages, grh/1 positive cells contained normal or only moderately reduced levels of nuclear DNp63 (Fig. 2A). At late somitogenesis, however, DNp63 had disappeared (Fig. 2B, white arrow) from grh/1-
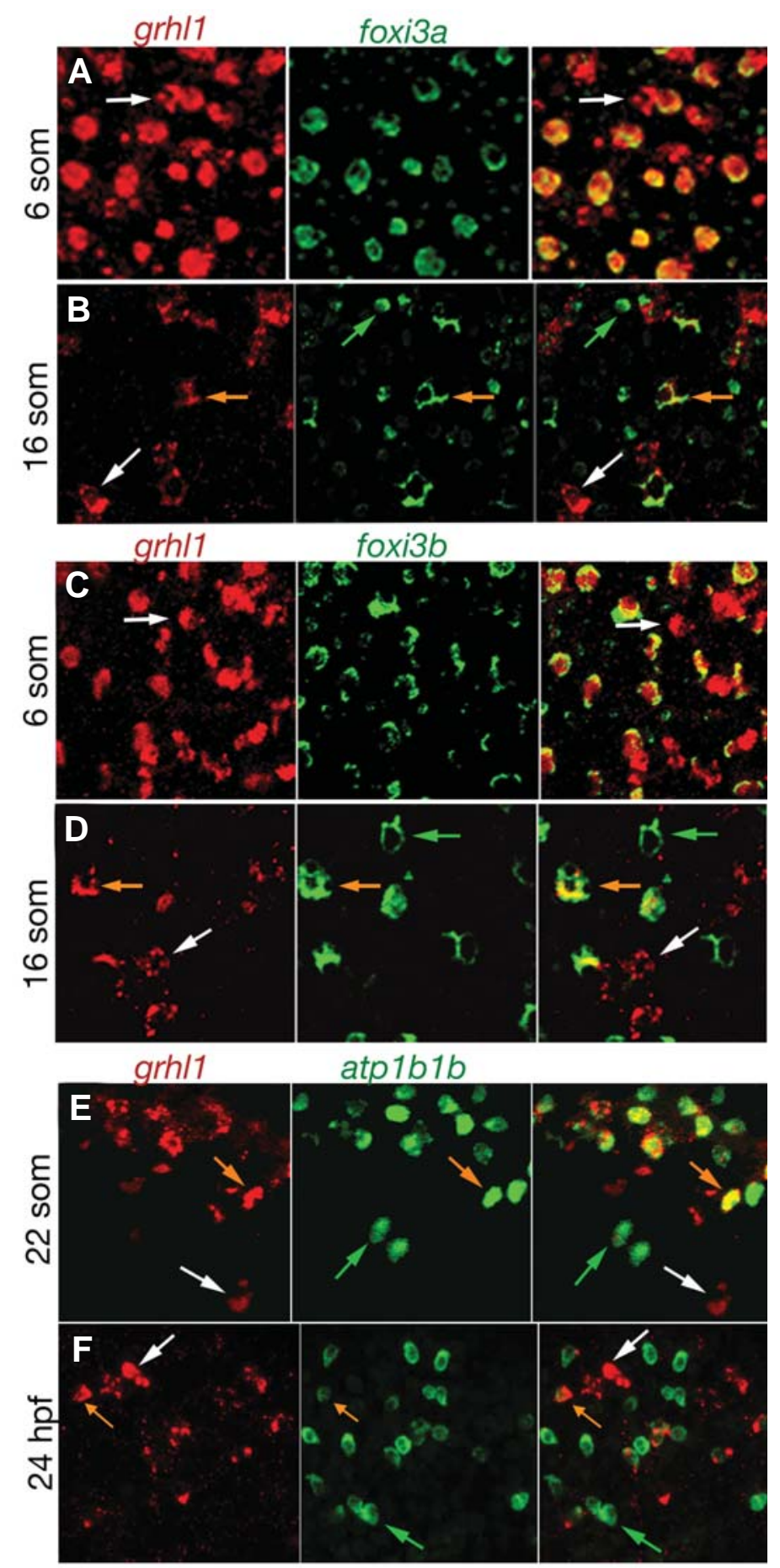

positive cells, or was present at much lower levels than in the grh/1-negative presumptive keratinocytes (Fig. 2B, orange arrow). Consistently, and in contrast to p63, grh/1 expression was absent from differentiated basal keratinocytes at $24 \mathrm{hpf}$ (30somite stage), as revealed by double fluorescent in situhybridizations for grh/1 and the keratinocyte markers collagen 1a1 (col1a1; Fig. 2 C,E). Together, this indicates that $g r h / 1$ labels nonkeratinocyte progenitors of the non-neural ectoderm.

To further investigate the identity of grh/1 expressing cells, we analysed its expression in comparison to that of ATPase genes, 
markers for differentiated ionocytes, and of foxi3 genes, which mark differentiated ionocytes as well as their progenitors. At the 6-somite stage all foxi3a and foxi3b cells expressed grh/1 (Fig. 3 A,C), while some grh/1-positive, foxi3-negative cells could also be detected (Fig. 3 A,C, white arrow). Such grh/1 single positive cells were also observed at the 16-somite stage (Fig. 3 B,D, white arrow). They could either represent less advanced ionocyte precursors prior to the onset of foxi3expression, or another, foxi3independent non-keratinocyte epidermal cell type. In contrast to the 6-somite stage, however, 16-somite stage embryos also contained foxi3-positive, but grh/1-negative cells (Fig. 3 B,D, green arrows) in addition to foxi3, grh/1 double positive (Fig. 3 $\mathrm{B}, \mathrm{D}$, orange arrows) and $g r h / 1$ single positive cells (white arrows). These foxi3single positive cells might represent more advanced, differentiating ionocytes, suggesting that in contrast to foxi3 genes, $g r h / 1$ is switched off during ionocyte differentiation. Corresponding temporal shifts in co-expression were observed for grh/1 and ATPase genes. At the 22-somite stage (20 hpf), approximately $36 \%(34.2 \pm 2.18 / 93.3 \pm 5.14, \mathrm{n}=5$ sections $)$ of $\mathrm{NaR}$ cells labelled by atp $1 b 1 b$ mRNA were also positive for grh/1 (Fig. $3 \mathrm{E}$, orange arrow). In contrast, at the 30 -somite stage (24 hpf) only approximately $18 \%$ of NaR cells (19.5 $\pm 3.25 / 109.2 \pm 3.76$, $\mathrm{n}=10)$ and $8 \%$ of $\mathrm{HR}$ cells $(5.0 \pm 1.14 / 62 \pm 2.86 ; \mathrm{n}=10)$, characterised by atp6v1al, expressed grh/1 (Figs. $3 \mathrm{~F}$ and S7C, orange arrows; Fig. $6 \mathrm{~N}$ ), while the number of $\mathrm{NaR}$ cells lacking grh/1 expression had increased accordingly (Fig. 3 E,F, green arrows; Fig. 6N). At both 20 and $24 \mathrm{hpf}$, however, embryos contained comparable numbers of $g r h / 1-$ positive, ATPasenegative cells, representing either ionocyte precursors and/or another non-epidermal cell type (Fig. 3 E,F, white arrows; Fig. 6N) (20 hpf: 63\%, 56.3 $\pm 3.35 /$ 89.3 $\pm 4.24, n=5 ; 24$ hpf: $\sim 54 \%, 31.0 \pm 3.41 / 57.2 \pm 3.50, n=10)$.

grhl1 is expressed in pvalb8-positive cells, which are distinct from $\mathrm{NaR}$ and HR ionocytes, but which also derive from the common pool of epidermal progenitors

To further analyse the nature of the grh/1-positive, but foxi3and ATPase negative cells, we studied the expression of pvalb 8

Fig. 4. pvalb8-expressing cells are distinct from $\mathrm{NaR}$ and HR ionocytes, but also display transient grhl1 expression and derive from the common pool of epidermal progenitors. All panels show in situ hybridisations and/or immunostainings with the probes and antibodies indicated in lower right corners. (A,B) Double fluorescent in situ hybridisations of wild-type embryos at 24 hpf, revealing that pvalb8positive cells lack expression of atp1b1b (A) and atp6v1al (B). (C) Double fluorescent in situ hybridisations of wild-type embryos at $24 \mathrm{hpf}$, revealing co-expression of grhl1 and pvalb8 in cells indicated by orange arrows. The left and middle pictures of each panel show the single channels of the merged image shown in the right picture. (D-F) Clones of epidermal cells of wild-type embryos at $24 \mathrm{hpf}$, after homotopic and homochronic transplantation of single GFP-transgenic cells into the ventral ectoderm at 6 hpf (shield stage). The left pictures of (D,E) show an anti-p63 immunostaining of keratinocytes (in blue), the right pictures show an overlay of the p63 immunostaining with an in situ hybridisation for pvalb8 transcripts (in red) and an anti-GFP immunostaining for the descendants of the transplanted cell (in green). The white arrows point to p63, pvalb8double negative cells in the clone, which might represent an NaR or HR ionocyte. (F) From left to right: in situ hybridization for pvalb8 transcripts (in red), in situ hybridization for atp1b1b and atp6v1al transcripts (in pink), anti-GFP immunostaining for the descendants of the transplanted cell (in green), merged image. (initially termed pvalb3a, but renamed according to http://zfin.org), which according to Hsiao et al. labels epidermal mucous cells (Hsiao et al., 2002). Indeed, in our fluorescent double in situ hybridisations, pvalb8, atp $1 b 1 b$ and atp6v1a/displayed complementary punctate expression patterns in $24 \mathrm{hpf}$ wild-type embryos (Fig. 4A,B). Carrying out grh/1, pvalb8double labellings, we further found that at $24 \mathrm{hpf}$, approximately $3 \%(1.7 \pm 0.4 / 57.2 \pm 3.5$, $\mathrm{n}=10$ ) of the grh/1-positive cells also displayed expression of pvalb8 (Figs. $4 \mathrm{C}$ and $6 \mathrm{~N}$ ), suggesting that similar to ionocytes, $g r h / 1$ is transiently expressed in the progenitors of pvalb8 cells.

We have previously shown that $\mathrm{NaR}$ and $\mathrm{HR}$ ionocytes derive from the same pool of epidermal progenitors as the keratinocytes (Jänicke et al., 2007). To test whether this is also true for pvalb\&expressing cells, the other grh/1-positive subpopulation of nonkeratinocyte cell types, we carried out similar cell lineage analyses, transplanting single GFP-labeled cells of the ventral (epidermal) ectoderm of an early gastrula embryo ( $6 \mathrm{hpf}$ ) homotopically into an unlabelled host of the same age, and staining the derivatives of the transplanted cell at $24 \mathrm{hpf}$ for p63 protein (keratinocyte
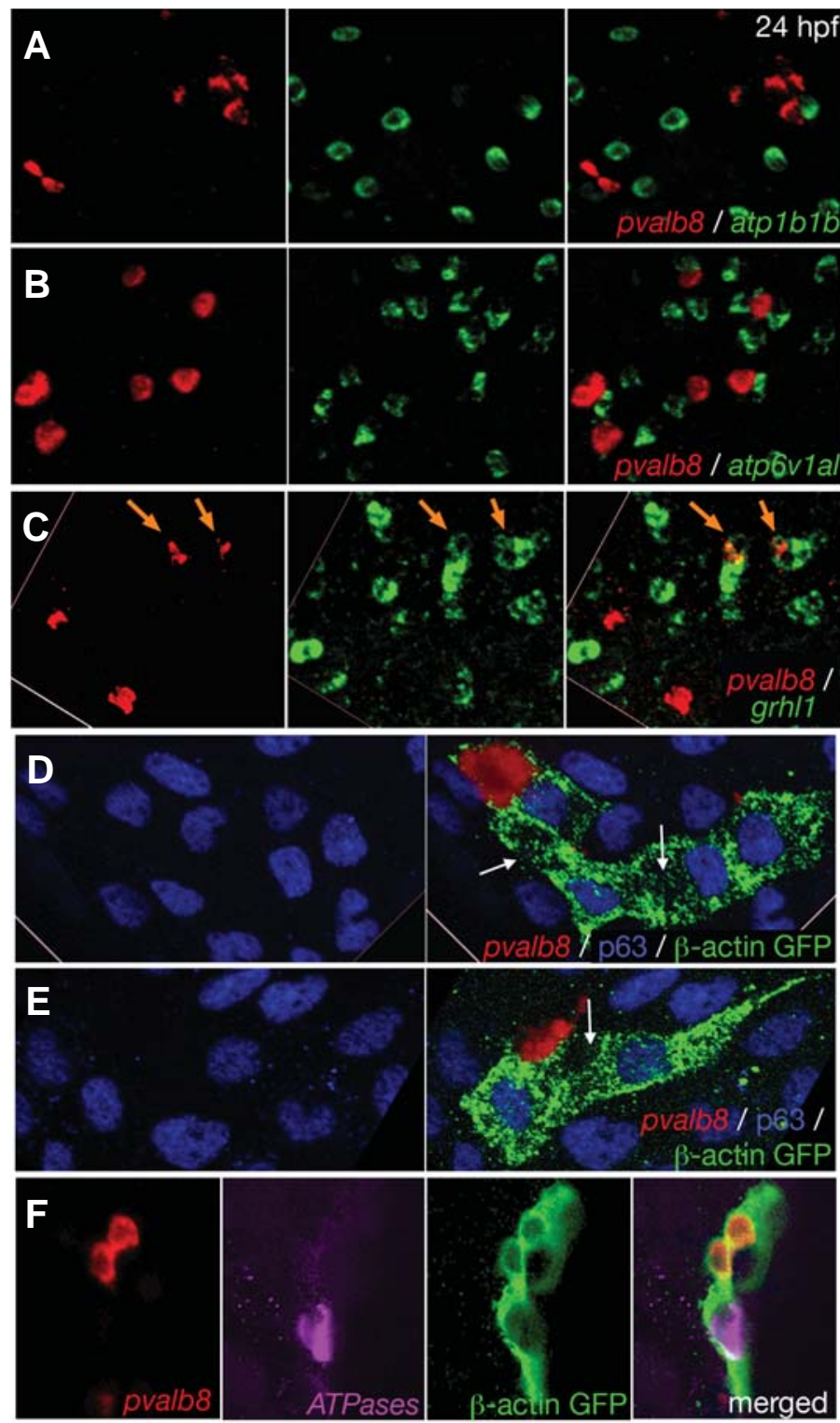

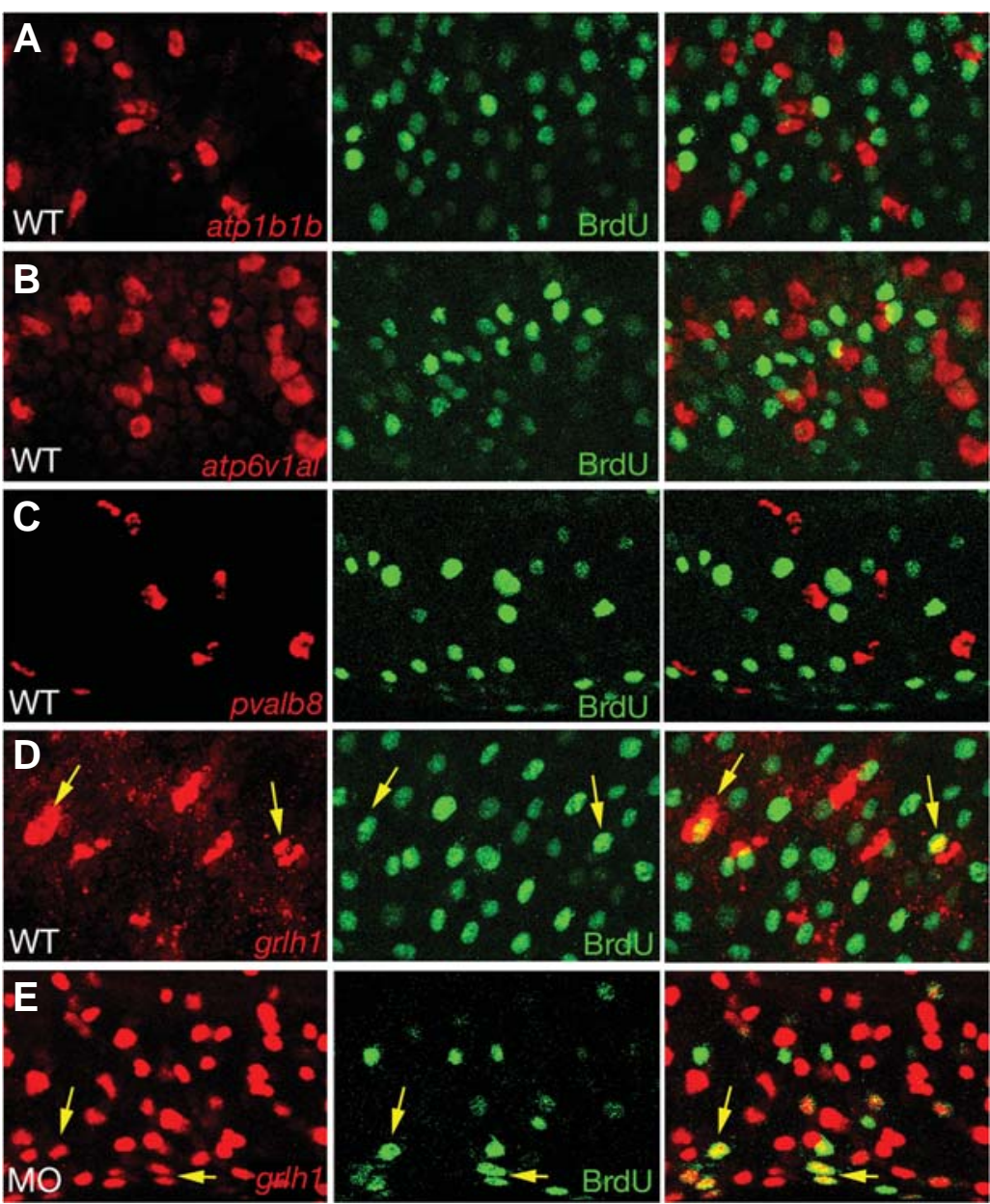

Fig. 5. grhl1 is expressed in proliferating cells. All panels show confocal sections of embryos at $24 \mathrm{hpf}$ that had been incubated with BrdU from $16-24 \mathrm{hpf}$, and after fluorescent in situ hybridization with probes indicated in red (left panels), and after anti-BrdU immunostaining (middle panels); right panels show merged images. (AD) Wild-type embryos; (E) grhl1 morphant injected with splice-MO (compare with Fig. 6 and Supplementary Fig. S7). Yellow arrows in (D,E) point to grhl1, BrdU-double positive cells. Differentiated ionocytes and pvalb8-positive cells lack BrdU incorporation (A-C), whereas over one quarter of grhl1-positive cells have undergone DNA replication between 16 and $24 \mathrm{hpf}$ (D) $(<28 \%, 17.6 \pm 2.83 / 62 \pm 4.45, n=10$ sections). (E) In morphant embryos, the absolute number of grhl1-positive cells increases enormously (see also Fig. 6N), whereas the absolute number of grhl1, BrdU-positive cells remains similar (19.3 $\pm 2.44 / 341 \pm 8.91,<5.5 \%, n=10$ sections), suggesting that the extra grhl1 cells represent differentiated non-keratinocyte epidermal cells, rather than their precursors.

marker) and pvalb8 mRNA. Clusters of four to eight cells were detected that consisted of one pvalb8 cell, several keratinocytes and one to two p63, pvalb8double-negative cells (Fig. $4 \mathrm{D}, \mathrm{E} ; \mathrm{n}=5$ / 5 clones). Double in situ hybridization further revealed the coexistence of pvalb8 and atp 1 b1b/atp6v1al-positive cells in such GFP-positive clusters (Fig. 4F; 3 clones). These finding show that epidermal pvalb8 cells derive from the ventral ectoderm and share the same progenitors as keratinocytes and ionocytes.

In contrast to differentiated ionocytes and pvalb8-positive cells, grhl1-positive cells undergo cell proliferation

To further look into the notion that $g r h / 1$ is expressed in precursors of, rather than fully differentiated nonkeratinocyte skin cells, we carried out BrdU incorporation studies to label cells undergoing DNA replication, in combination with in situhybridization for grh/1, pvalb8or ATPase transcripts. Interestingly, we found that grh/1positive cells do not only incorporate BrdU during early segmentation stages ( $10 \mathrm{hpf}-16 \mathrm{hpf}$; data not shown), when the first ionocytes are formed, but also during midsegmentation stages (15 hpf -24 hpf; Fig. 5D). In contrast, ATPase or pvalb8-positive cells of sibling embryos treated during the same mid-segmentation interval were all BrdU-negative (Fig. 5 A-C). Together with the aforementioned lineage tracing and co-expression analyses, these findings indicate that during the first day of development $g r h / 1$ is temporarily expressed in proliferating progenitors of a number of different, nonkeratinocyte skin cells that derive from the same pool of epidermal precursor cells, while expression is downregulated during cell differentiation.

\section{Grhl1 is dispensable for proper specification and function of the different skin cells}

Loss-of-function studies in Drosophila, Xenopus and mouse have identified various roles for grainyhead and its vertebrate homologues within different developmental processes such as epidermal specification (Bray and Kafatos, 1991; Ting et al., 2003; Tao et al., 2005; Chalmers et al., 2006; Yu et al., 2006), duct maturation of the salivary gland and the kidney (Yamaguchi et al., 2006), as well as position-specific differentiation of Drosophila postembryonic neuroblasts ( $\mathrm{pNBs}$ ), influencing timing and duration of proliferation (Almeida and Bray, 2005; Cenci and Gould, 2005; Maurange et al., 2008). In thoracic pNBs grainyhead prevents premature cell-cycle exit by repressing prospero, a homeodomain protein. Therefore, premature as well as increased expression of prospero can be observed in grh mutants (Maurange et al., 2008).

To understand the function of $g r h / 1$ in the zebrafish embryo, we analysed the loss-of-function phenotype generated by injection of specific antisense morpholino oligonucleotides (MOs) into the 1-2-cell stage embryo. Two different MOs targeting either the initiation of translation (grh/1-ATG-MO) or correct splicing of the gene (grh/1-splice-MO) were used. At the concentrations used, the ATG-MO completely blocked translation of co-injected mRNA containing the $\mathrm{MO}$ binding site fused upstream of GFP (Supplementary Fig. S1 A-C). The splice-MO blocked correct splicing of the fourth intron, which introduced a premature stop codon before the DNA binding CP2 domain (Supplementary Fig. S1 D-E). In the experiments described below, the ATG and splice MOs were either injected alone or together, and with or without p53 MO to suppress unspecific MO-induced apoptosis (Robu et al., 2007). However, in all conditions, identical results were obtained (Supplementary Fig. S2), suggesting that both morpholinos generated amorphic phenotypes.

The zebrafish homologue of Drosophila prospero(see above), 
prospero-related-homeobox-gene-1 (prox 1) is expressed in non-keratinocyte skin cells at $1 \mathrm{dpf}$ (Thisse, 2001; http://zfin.org). However, both in grh/1 morphants and un-injected controls, its expression started at the 28-somite stage, and no difference in cell numbers or staining intensity was observed at $24 \mathrm{hpf}$ (Supplementary Figs. S3 G,H and S4 D,I). In addition, the spatial and temporal expression patterns of the three aforementioned non-keratinocyte skin cell markers atp1b1b, atp6v1al and pvalb8 appeared unaltered in grh/1 morphants (Supplementary Fig. S3 A-F and S4 A-C,I). Furthermore, we found normal numbers of $n c \times 1 b$ - and trpv6 (previously called ecac)expressing cells, representing subsets of NaR cells (Pan et al., 2005; Liao et al., 2007; Hwang and Lee, 2007) (Supplementary Fig. S4 E,F,I). Recently, the existence of at least two other skin cell populations, termed NCC (thiazide-sensitive $\mathrm{Na}^{+} / \mathrm{Cl}^{-}$
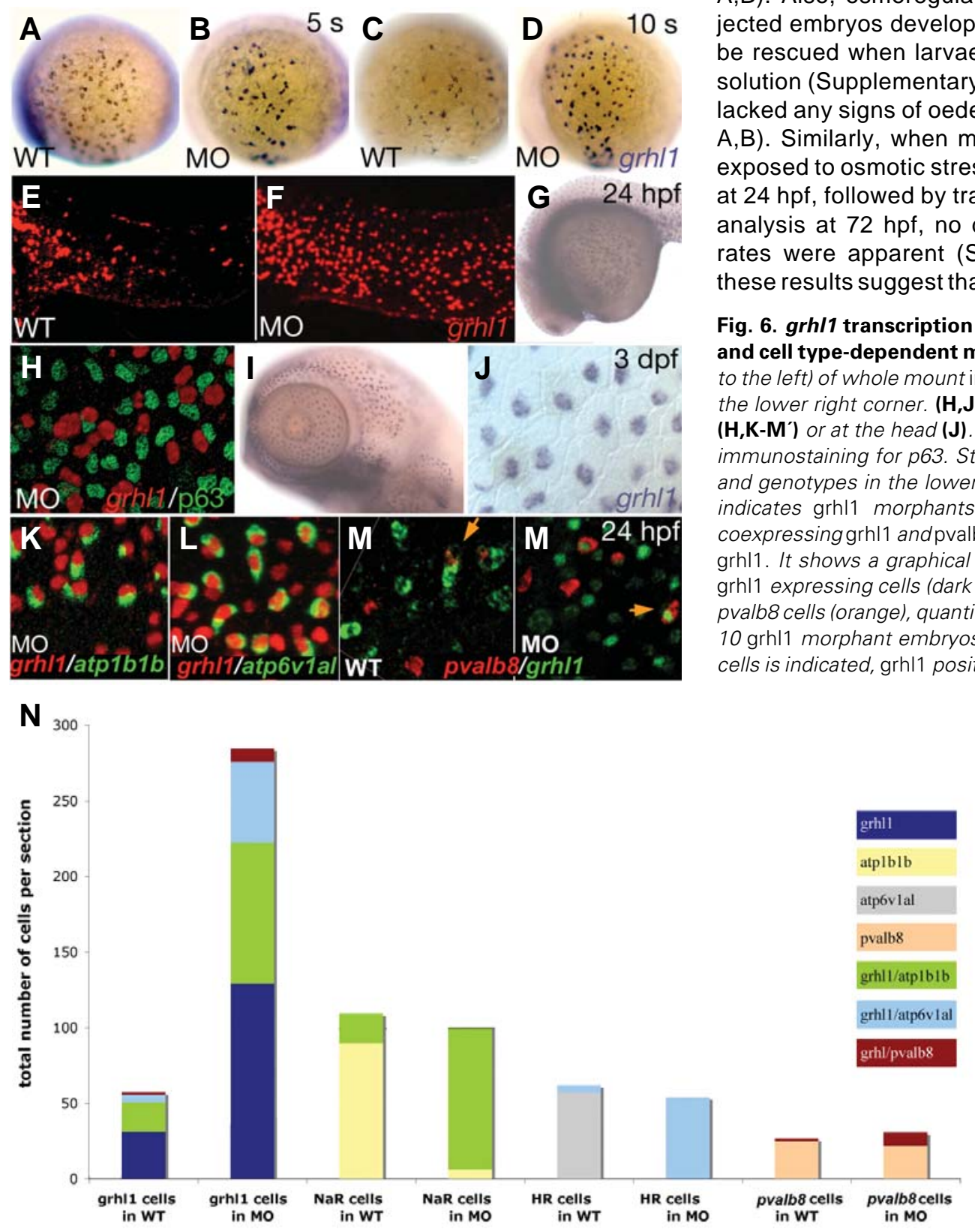

Fig. 6. grhl1 transcription is controlled in an autoregulatory, timeand cell type-dependent matter. (A-M') Lateral views (anterior/ventral to the left) of whole mount in situ hybridisations with probes indicated in the lower right corner. (H,J,K-(M') Cells at the anterior end of the trunk $\left(\mathbf{H}, \mathbf{K}-\mathbf{M}^{\prime}\right)$ or at the head $(\mathbf{J})$. (H) In situ hybridisation was followed by an immunostaining for p63. Stages are indicated in the upper right corner and genotypes in the lower left corner and apply to all panels left. MO indicates grhl1 morphants. Orange arrows in (M, $\mathbf{M}$ ') indicate cells coexpressing grhl1 and pvalb8. (N) Coexpression of skin cell markers and grhl1. It shows a graphical illustration of the average total numbers of grhl1 expressing cells (dark blue), NaR cells (yellow), HR cells (grey) and pvalb8 cells (orange), quantified for the left side of the trunk of 10 WT and 10 grhl1 morphant embryos at $24 \mathrm{hpf}$. The proportion of co-expressing cells is indicated, grhl1 positive NaR cells (green), grhl1 positive HR cells (light blue) and grhl1 positive pvalb8 cells (dark red). It can be seen that total numbers of ionocytes and pvalb8 cells do not change between WT and morphants, while the proportion of co-expressing cells increases. Please note that in the grhl1-splicemorphants shown in this figure, the grhl1 mRNA was located primarily in the nuclei. This is in contrast to the cytoplasmic localisation of other mRNAs, see (K-M'), and in contrast to grhl1 mRNA in wild-type (see Fig. 2A) or in grhl1-ATG-MO-injected embryos (compare Supplementary Fig. S5C with S5D). Since splicing of hnRNA occurs in the nucleus, incorrectly or not fully spliced grhl1 transcripts appear to be incapable of nuclear export. Apart from this different RNA localisation, identical results with progressively increasing numbers of grhl1-positive cells were obtained for both grhl1-ATG$\mathrm{MO}$ and grhl1-splice-MO. 


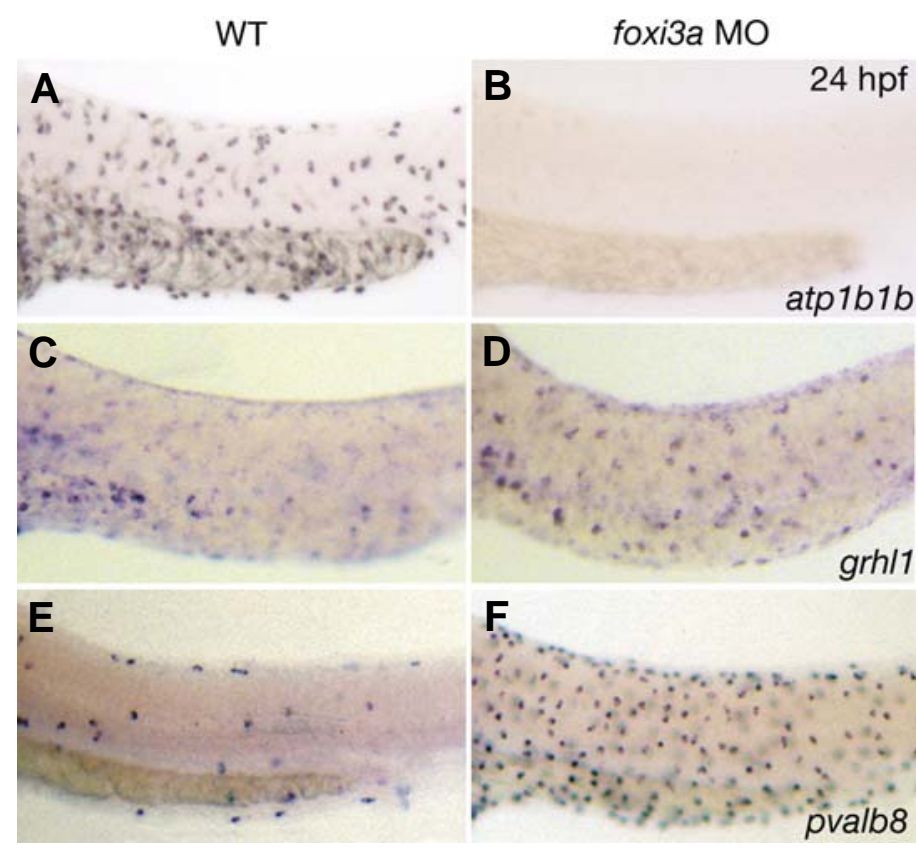

Fig. 7. Loss of foxi3a function causes loss of ionocytes and an expansion of pvalb8-positive cells, while the number of grh/1positive cells remains unaltered. All panels show whole mount in situ hybridisations at $24 \mathrm{hpf}$ with probes indicated in the lower right corner; $(\mathbf{A}, \mathbf{C}, \mathbf{E})$ un-injected controls; (B, D,F) foxi3a morphants. Anterior is to the left, dorsal up.

development and function of the different skin cell types.

\section{grhl1 is required for negative transcriptional autoregula- tion in differentiating ionocytes}

In contrast to all other investigated skin markers mentioned above, grh/1 morphants displayed progressively higher numbers of cells positive for grh/1 itself. At the 5 -somite stage, numbers of dispersed grh/1-positive epidermal cells in grh/1 morphants were still normal (Fig. 6 A,B). However, at the 10 somite-stage, cell numbers in morphants were already approximately double as high as those of control embryos (Fig. $6 \mathrm{C}$ and $D)$, while at $24 \mathrm{hpf}$, the increase was up to five-fold (6E,F and $N)$. In addition to the increase in the number of the dispersed epidermal cells, expression within the periderm cells was stronger and clearly detectable in the entire skin (Fig. 6G). This expression was prolonged until $3 \mathrm{dpf}$ (Fig. $6 \mathrm{I}, \mathrm{J})$. None of the additional $\mathrm{grh} / 1$ cells expressed $\mathrm{p} 63$, indicating that they are no keratinocytes from the basal layer (Fig. $6 \mathrm{H}$ ). In reverse, prominent $g r h / 1$ expression in non-keratinocyte epidermal cells could be largely suppressed upon grh/1 mRNA injection, while coexpression of $g r h / 1 \mathrm{mRNA}$ and $g r h / 1 \mathrm{MO}$ led to a compensation of the two opposite effects, and to wild-type conditions (Supplementary Fig. S6).

The increase in grh/1positive single cells in grh/1 morphants starts around the same time when the co-expression of foxi3 and $g r h / 1$ begins to decrease in wild-type embryos. This suggest that under wild-type conditions, grh/1 is down-regulated in ionocyte progenitors by an autoregulatory mechanism during ionocyte differentiation, while this negative feedback is inhibited upon knockdown of the gene. Consistent with this notion, despite the massive increase in the number of grh/1-positive cells, the absolute number of BrdU-positive, grh/1-positive cells of $g r h / 1$ morphants at $24 \mathrm{hpf}$ remained as in uninjected controls (Fig. $5 \mathrm{D}, \mathrm{E}$ ), indicating that the supernumerary grh/1-positive cells represent differentiated non-keratinocyte skin cells, rather than additional progenitors of the this lineage. Consistent results were obtained via grh/1-ATPases double stainings of differentiated ionocytes. While in wild-type embryos, $18 \%$ of the $\mathrm{NaR}(19.5 \pm 3.25 / 109.2 \pm 3.76, \mathrm{n}=10)$ and $8 \%$ of HR $(5 \pm 1.14$ / $62.0 \pm 2.86, \mathrm{n}=10$ ) cells expressed grh/1 at $24 \mathrm{hpf}$, this proportion increased in grh/1 morphants to $94 \%(93.25 \pm 2.87$ / $99.5 \pm 1.65, \mathrm{n}=10)$ and $99 \%(0.6 \pm 0.4 / 54.2 \pm 2.26, \mathrm{n}=10)$, respectively (compare Fig. $6 \mathrm{~K}$ with Fig. 3F; see also Fig. $6 \mathrm{~L}, \mathrm{~N}$ and Supplementary Fig. S7 C-D). However, the overall numbers of both types of ionocytes remained constant, indicating that $g r h / 1$ was up-regulated in these cells rather than additional cells being formed (Fig. $6 \mathrm{~N}$ ). In contrast to ionocytes, the majority of pvalb8 expressing cells remained grh/1 negative even in grh/1 morphants, although an increase of co-expressing cells from roughly $7 \%$ in wild types $(1.7 \pm 0.4 / 26.7 \pm 2.46, n=10)$ to $28 \%$ in morphants $(8.8 \pm 2.92 / 30.8 \pm 2.65, \mathrm{n}=10)$ could be detected (Fig. $\left.6 \mathrm{M}, \mathrm{M}^{\prime}, \mathrm{N}\right)$. The presence of $g r h / 1$-negative pvalb8 cells suggests that in this cell-type, and in contrast to ionocytes, grh/1 expression can be switched off via another mechanism that acts in parallel to the described negative Grhl1 autoregulation.

\section{foxi3a morphants display normal numbers of grhl1-, but significantly increased numbers of pvalb8-positive cells}

The data described thus far indicate the presence of different non-keratinocyte skin cell lineages, all of which derive from the same pool of epidermal precursors, and all of which display transient expression of grh/1. In contrast, expression of foxi3a and foxi $3 b$ is restricted to a subset of this population, driving the specification of cells towards the $\mathrm{NaR}$ and $\mathrm{HR}$ ionocyte fate. Knockdown of foxi3 leads to a loss of ionocytes, indicated by the absence of atp 1b1band atp6r1a/expression at $24 \mathrm{hpf}$ (Fig. 7 A,B; Hsiao et al., 2007; Jänicke et al., 2007). In contrast, knockdown of foxi3aor foxi3bor bothdid not affect the numbers of grh/1 positive cells at $24 \mathrm{hpf}$ (Fig. 7 C,D and data not shown). Most strikingly, pvalb8-expressing cells even displayed a positive response, opposite to that of $\mathrm{NaR}$ and HR ionocytes, with significantly increased numbers in foxi3a morphant embryos at $24 \mathrm{hpf}$ (Fig. $7 \mathrm{E}, \mathrm{F}$ ). These findings are in line with the notion that grh/1 is a common marker for both Foxi3-promoted ( $\mathrm{NaR}$ and $\mathrm{HR}$ ) and Foxi3-suppressed (pvalb8-positive) cells, and that Foxi3a may act as a molecular switch converting cells that would otherwise express pvalb8 to $\mathrm{NaR}$ or HR ionocytes.

\section{Discussion}

The Grh/CP2 family of transcription factors is widely conserved within the animal kingdom and has been implicated in many developmental pathways such as terminal differentiation of the skin and neurogenesis (Bray and Kafatos, 1991; Cenci and Gould, 2005; Tao et al., 2005; Maurange et al., 2008). In this study, we have analysed the conservation of the family in the zebrafish genome and investigated the expression and function of $z-g r h / 1$ in the zebrafish skin, with special focus on non-keratinocyte cell lineages and the relationship between 
Grhl1 and the known ionocyte regulator Foxi3. To our knowledge, no Grh/CP2 member has been described in this context in any organism before.

\section{Seven members of the Grh/CP2 family exist within the zebrafish genome}

During early vertebrate evolution, two rounds of whole-genome duplication are thought to have occurred, generating four homologues of each invertebrate gene, some of which were subsequently lost (reviewed in Meyer and Schartl, 1999). Consistent with this model the Grh/CP2 family has expanded from one gene, $\mathrm{Ce}$ grh1, in C. elegans, and two members, D-grh and D-Cp2, in Drosophila, to six homologues, named Grh/1-3, Cp2, Cp2-like1 and $\angle b p-1 a$ in mammals (Bray and Kafatos, 1991; Wilanowski et al., 2002; Venkatesan et al., 2003).

In the teleost branch, a third round of whole-genome duplication took place, although many of duplicated genes were not maintained during further evolution (Postlethwait et al., 1998). We have identified seven homologues of $D$-Grh/Cp2 within the zebrafish genome (Fig. 1). Two of those, grh/1 and z-cp2, had been previously proposed based on partial EST sequences (Venkatesan et al., 2003). Upon alignment with published invertebrate and vertebrate Grh//Cp2 genes, the seven zebrafish genes clustered with the six known mammalian groups described above (Fig. 1). The fact that no seventh or eighth group was identified confirms that the radiation of the invertebrate grainyheadand Cp2gene had already occurred in the ancient vertebrate lineage prior to the split of the actinopterygia, ray-fined-fish, and the sarcopterygian, which include tetrapodes and lungfish. Our analysis further suggests that most paralogues of the grh/ CP2genes generated during the third round of genome duplication were not maintained in the zebrafish lineage, as only one duplicated pair was found, grh/2a and grh/2b. These two paralogue genes show different expression patterns, which indicates that they may have adopted different functions and therefore were both subject to selective pressure and hence were retained. While grh/2b is ubiquitously expressed, expression of grh/2a is restricted to the olfactory and otic placodes as well as to the developing pronephros (Fig. 1). The mouse homologue, grh/2, is also expressed in the developing kidney (Auden et al., 2006; Yamaguchi et al., 2006) indicating a potential conserved role of grh/2 during kidney development.

\section{Zebrafish grhl1 and grhl3 are transiently expressed in the periderm}

In Xenopus and mouse, members of the Grhl subfamily are involved in skin development. In Xenopus, expression of Grh/1and Grh/3is found in the non-neural ectoderm and later in the superficial layer of the epidermis (Tao et al., 2005; Chalmers et al., 2006), which was proposed to be a homologous structure to the zebrafish periderm (Sagerstrom et al., 2005). Within the skin of mice Grh/1 and Grh/3are expressed in all suprabasal layers, which are formed by terminally differentiating cells deriving from the basal cells, and detected from day E8.5 with progressively increasing expression. In contrast, Grh/2expression also starts at E8.5 but decreases until $\mathrm{E} 12.5$, and is restricted to the basal layer, the stratum spinousum and the stratum granulosum (Auden et al., 2003).

Of the two epithelial layers of the zebrafish epidermis, $g r h / 1$ is only expressed in the outer periderm, but not in the basal layer (Fig. 1 ), similar to the situation in Xenopus, and similar to the basal exclusion of $G r h / 1$ in mouse. Furthermore, the zebrafish periderm displays at least temporary co-expression of $g r h / 1$ with $g r h / 3$, although the temporal expression profiles of the two genes appear quite different, with an earlier onset and cessation of $g r h / 3 e x p r e s-$ sion. This temporal co-expression of the two paralogs in the zebrafish periderm again is very similar to the behaviour of their orthologs in the outer skin layer in Xenopus (Tao et al., 2005; Chalmers et al., 2006) and in the suprabasal layers in mouse (Auden et al., 2003).

\section{grhl1 is transiently expressed in specifying ionocytes}

In addition to the periderm, zebrafish grh/1 is highly expressed in single cells spread across the basal epidermis in a punctate pattern, starting at early segmentation stages (Fig. 1). Several lines of evidence suggest that some of these cells are precursors of $\mathrm{NaR}$ and HR-type ionocytes. We and others have previously shown that precursors of these ionocyte types express foxi3a and foxi3b, which encode transcription factors required for ionocyte differentiation and possibly ATPase gene activation (Jänicke et al., 2007; Hsiao etal., 2007). During early segmentation stages (up to the 10somite stage), all foxi3-positive cells co-express grh/1 (but not all grh/1-positive cells co-express foxi3a, see below). However, during further development, more and more foxi3-positive, but grh/1negative cells show up, suggesting that $g r h / 1$ is switched off during early steps of ionocyte differentiation (Fig. 3). Accordingly, wildtype larvae display only a few cells with grh/1/atp1b1b or grh/1/ atp6v1a/coexpression (18\% of $\mathrm{NaR}$ cells and $8 \%$ of $\mathrm{HR}$; Figs. 3 and 6). The co-existence of foxi3/ grh/1 and ATPase/grh/1 doublepositive cells together with foxi3 and ATPase -positive cells lacking $g r h / 1$ transcripts provides further support to our previously proposed model that the neo-formation of ionocytes is a continuous process (Jänicke et al., 2007). Consistently, we have shown here that grh/1-positive cells can proliferate and incorporate BrdU even during mid segmentation stages, in co-existence with postmitotic, fully differentiated non-keratinocyte cell types that are all BrdU-negative (Fig. 5). Important further evidence for a transient grh/1 expression in all $\mathrm{HR}$ and $\mathrm{NaR}$ ionocytes comes from our analysis of $g r h / 1$ morphants (Fig. 6). Although other, more complicated interpretations cannot be completely ruled out, these data strongly suggest that Grhl1 protein is required for the downregulation of $g r h / 1$ transcription during ionocyte differentiation. Upon knock-down of $g r h / 1$, grh/1 transcription is maintained and can be used as a lineage tracer, now marking almost all $\mathrm{NaR}$ and HR type ionocytes even during later stages of development (compare Fig. 3 with Fig. 6, and see Fig. $6 \mathrm{~N}$ for quantification). To our knowledge, this is the first report of a negative autoregulative mechanism for any grh/CP2 family member and in any organism. Such regulatory feedback loops seem a very common regulative mechanism during vertebrate development, and been shown for multiple other zebrafish transcription factors such as Hoxb1a, Pax6 or Gata1 (McClintock etal., 2002; Aota etal., 2003; Kobayashi et al., 2001). Future studies have to show whether the negative Grhl1 autoregulation is direct or mediated by other transcription factors. Interestingly, sequence analysis of the $g r h / 1$ gene revealed a $33 \mathrm{bp}$ DNA element approximately $5 \mathrm{~kb}$ upstream of the transcriptional start site with $60.6 \%$ identity (20/33) with a previously described essential Grhl1 binding site in the Xenopuskeratin gene XK81A1 (Huang et al., 1995; Tao et al., 2005) (Supplementary Fig. S8 A-C). This upstream region, as well as the putative 


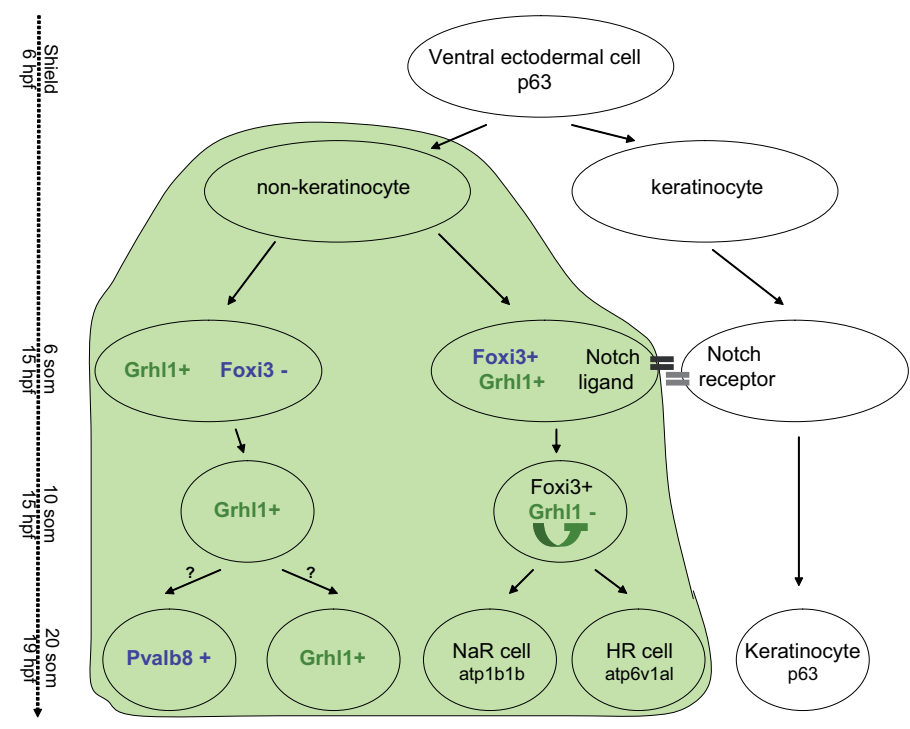

Fig. 8. Model summarising known and proposed mechanisms driving the sequential segregation of different epidermal cells types of the zebrafish embryo. This model is based on a combination of previously described data (Hsiao et al., 2007; Jänicke et al., 2007), and data presented here (highlighted in bold). The developmental time points are indicated on the left. All non-keratinocyte epidermal cells labelled bygrhl1 are on a light green background. The mechanisms driving their segregation from the common pool of ventral ectodermal cells are not known, but might involve stochastic events. The Grhl1 lineage further segregates into a foxi3-positive and a foxi3a-negative sub-lineage. The mechanisms restricting the initiation of foxi3 expression to a subset of the grhl1 population remain unclear, however, the involvement of Notch signalling can be ruled out (see text for further discussion). Whether the foxi3negative grhl1 cells exclusively differentiate into pvalb8 cells or additional, thus far unknown epidermal cell types, remains unclear and is indicated by question marks (see text for further discussion). According to previous work, the Foxi3 lineage employs lateral inhibition via Notch signalling to prevent a further expansion of NaR/HR ionocytes at the expense of keratinocytes (Hsiao et al., 2007; Jänicke et al., 2007). This does not seem to be the case for pvalb8 cells, which according to our unpublished data do not respond to interferences with Notch signalling. The Foxi3 lineage, but not the non-Foxi3 lineage, further displays negative auto-regulation of grhl 1 expression. Whether this negative autoregulative autoregulation is required for $N a R / H R$ ionocyte differentiation is unknown.

promoter region, also contains multiple potential binding sites for Foxi1 (T(g/a)TTT(g/a)(t/c); Kurth et al., 2006), a close Foxi3 relative (Supplementary Fig. S8 A,D). Recently, zebrafish foxi1 has been shown to be required upstream of the foxi3genes for skin ionocyte development (Esaki et al., 2009). It will be interesting to elucidate the functional connection between foxit and grh/1. If Foxi3 proteins have a direct effect on grh/1transcription in ionocytes, it most likely is positive, rather than negative, as suggested by our unpublished observation according to which the punctate grh/1 expression is absent, rather than more persistent, in foxi3morphants.

\section{grhl1 is transiently expressed in other non-keratinocyte epi- dermal cell lineages}

In grh/1 morphant embryos with prolonged grh/1 expression, only approximately half of the grh/1 population was ATPasepositive and thus belonged to the ionocyte lineage. The exact nature and composition of the other half is not totally clear. Some of the cells might represent immature ionocytes (see above), while others belong to the pvalb8-lineage, indicated by $g r h / 1 /$ pvalb8 co-expression at 24 hpf (Figs. 4,8). pvalb8 has been previously described as a marker of epidermal mucous cells, an assumption that was solely based on its punctate epidermal expression pattern (Hsiao et al., 2002). Also, Pvalb8 itself does not allow definitive conclusions about the nature of the cells. It represents a $\mathrm{Ca}^{2+}$ binding protein that is thought to be a cytosolic ion reservoir modulating $\mathrm{Ca}^{2+}$-dependent signalling pathways (reviewed by Pauls et al., 1996; Zacchia and Capasso, 2008). However, our double in situanalyses show that pvalb8, atp $1 b 1 b$ and atp6v1al are indeed expressed in a mutually exclusive manner, identifying the pvalb8cells at least as a distinct epidermal population. Furthermore, according to our lineage tracing experiments, they derive from the same population of epidermal precursor cells like keratinocytes and ionocytes (Fig. 4). In this light, grh/1 appears as a more general marker of non-keratinocyte cell types, in contrast to foxi3aand foxi 36 , which are restricted to $\mathrm{NaR}$ and HR ionocytes (Fig. 8). Future experiments have to reveal whether $g r h / 1$ is also expressed in progenitors of further, thus far uncharacterized epidermal cell lineages. Such a lineage has been recently proposed by the Hwang laboratory and named NCC cells, while nothing is known about its embryonic origin, and no markers are available to stain cells prior to $3 \mathrm{dpf}$ (reviewed in Hwang and Lee, 2007; see Supplementary Fig. S4 of this study).

\section{Foxi3 plays a crucial role in subdividing the Grhl1 population by promoting $\mathrm{NaR} / \mathrm{HR}$ ionocyte specification while blocking the pvalb8 fate}

We and others have previously shown that Foxi3 is required for NaR/HR ionocyte differentiation (Hsiao etal., 2007; Jänicke etal., 2007). Here, we show that the loss of ionocytes in foxi3amorphants is accompanied by a gain of pvalb8 cells, while the number of cells expressing the common marker grh/1 remains unaltered (Fig. 7). This suggests that foxi3not only promotes the ionocyte fate, but also suppresses the pvalb8 fate, meaning that Foxi3 induces a switch from the "default" pvalb8to the NaR/HR ionocyte fate (Fig. 8). Similar fate-switching effects have been described for multiple other transcription factors, such as the Pou-homeodomain protein Pit1, which converts adenohypophyseal cells from their "default" gonadotrope or corticotrope to the somatotrope, lactotrope or thyrotrope fate by activation and repression of the corresponding hormone gene (Nica et al., 2004; and references therein).

Currently, we can only speculate about the mechanisms regulating the differential expression of foxi3genes in some, but not all of the $g r h / 1$ cells. However, it is interesting to note that at $24 \mathrm{hpf}$ the ratio of atp $1 \mathrm{~b} 1 \mathrm{~b} / \mathrm{grh} / 1$ and atp6v1al/grh/1 double positive versus ATPasenegative grh/1 cells is close to 50:50 (Fig. 6N), and that in our lineage tracing experiments, epidermal clones often contained two different non-keratinocyte ( $p 63$-negative) cell types next to each other, a pvalb\&-positive and a atp1b1b or atp6v1a/-positive cell (Fig. $4 \mathrm{D}-\mathrm{F}$ ). Thus, it is tempting to speculate that foxi3-positive and foxi3-negative grh/1 cells might have the same mother cells (Fig. 8). Consistent with this notion, we found that some of the grh/1-positive cells incorporated BrdU during mid-segmentation stages (Fig. 5), thus, after they might have been singled out from the pool of epidermal progenitors by the Notch system (Jänicke and Hammerschmidt, 2007). It is tempting 
to speculate that these rather late cell divisions might be the ones that give rise to a ionocyte and a pvalb8 cell from a singled-out non-kerationcyte precursor. During development, such binary cell fate determination processes are rather common mechanisms, most of which involve a combination of asymmetric localization of intrinsic factors in the mother cells, such as the Notch signalling modulators Numb and Neutralized or the aforementioned Prospero (Le Borgne and Schweisguth, 2003; Manning and Doe, 1999), and differential extrinsic signalling on sibling cells, e.g. via Notch or Epithelial Growth Factor (EGF) (Berset et al., 2005; Shin et al., 2007). We found normal numbers of pvalb8positive cells in mutants deficient for Notch signalling and after constitutive Notch activation (unpublished own data; compare with Jänicke et al., 2007), suggesting that such a binary pvalb8$\mathrm{NaR} / \mathrm{HR}$ cell fate decision within the grh/1 population of nonkeratinocyte epidermal cells is independent of the Notch system. More studies will be necessary to unravel the exact mechanisms underlying these sub-functionalisation events.

\section{grhl1 morphants do not show discernable defects}

Similar to the temporal expression profile described here for zebrafish grh/1, Drosophila grh displays transient expression in specific progenitor cells, e.g. in postembryonic neuroblasts (pNBs), while expression ceases when the cells stop to divide (Uv et al., 1997). Mutant analyses further revealed that $g r h$ is required for production of the correct number of pNBs, dependent on their temporal and spatial position within the larvae. While the lineage of thoraic pNBs decreased in mutants, the numbers of abdominal pNBs increased (Almeida and Bray, 2005; Cenci and Gould, 2005; Maurange et al., 2008). It is tempting to speculate that in zebrafish $\mathrm{grh} / 1$ functions in a similar way to its Drosophila homologue and is involved in correct timing of differentiation of nonkeratinocyte skin cell lineages.

However, knock-down of grh/1via injection of different antisense MOs did not yield any discernable defects in the timing of differentiation or the final numbers of the different non-keratinocyte epidermal cells (Supplementary Figs. S3-S5). Furthermore, in contrast to Xenopusand mouse Grh/1(Tao etal., 2005; Wilanowski et al., 2008), we found zebrafish grh/1 to be dispensable for keratinocyte development.

We can only speculate about the reasons for the dispensable nature of zebrafish grh/1. It is possible that another member of the family or a different transcription factor can compensate for loss of grh/1. Knockout of mouse CP2 does not lead to any obvious phenotype, and it was shown that the highly similar paralogue LBP-1 a can compensate for loss of CP2 in mouse and human cell culture (Ramamurthy et al., 2001; Kang et al., 2005). A candidate accounting for such functional redundancy in ionocytes and pvalb8cells of the zebrafish could be $g r h / 2 b$, which is ubiquitously expressed during the first days of development (Fig. 1). In the periderm, there could be functional redundancy between grh/1 and $g r h / 3$, which are coexpressed in this outer layer of the embryonic skin, but not in ionocyte and pvalb8 cell precursors (Fig. 1; see also above). grh/1 and grh/3 double knock-down experiments are in progress to test this possibility. In mouse, both Grhl1 and Grhl3 are essential for skin development (Tao et al., 2005; Ting et al., 2005; Hislop et al., 2006; Yu et al., 2006; Yu et al., 2008). This suggests that during vertebrate evolution, the two paralogs might have given up their initially redundant roles to fulfil specialised functions in the more complex mammalian skin.

Whether the observed indispensable role of Grhl1 as a negative auto-regulator of its own expression in non-keratinocyte epidermal cells is of biological relevance is unclear, since in our morphant animals, the persistently generated grh/1 transcripts are inactive. To look into this, it would be necessary to force prolonged $g r h / 1$ expression in differentiating epidermal cells. One attractive possibility consistent with some of the fly data would be that such prolonged expression leads to delayed ionocyte differentiation and/or further progenitor proliferation. Our preliminary experiments with grh/1 mRNA injection did not have any effects apart from a reduction of endogenous grh/1transcription (Supplementary Fig. S6; and unpublished observations). However, this might be due to insufficient stability of the injected RNA (up to early segmentation stages; Harland and Misher, 1988; Fink et al., 2006), and does not necessarily mean that the observed downregulation of $g r h / 1$ expression in differentiating ionocytes is dispensable.

\section{Materials and Methods}

\section{$R T-P C R$}

foxi3a, foxi3b, grhl1, grh/2a, grhl2b, grh/3, cp2, cp2-like1, Ibp-1a, prox $1, n c \times 1 b$, and $n c c 22 b c D N A$ fragments were amplified via RT-PCR with total RNA isolated from zebrafish embryos at 12, 24, 48 and / or 72 hpf, and the primers listed in Supplementary Table 1.

\section{Labelling procedures}

Whole mount in situ hybridisations and immunostainings were performed as previously described (Hammerschmidt et al., 1996). In situ hybridizations for each $\mathrm{grh} / \mathrm{gene}$ were carried out with probes of two different lengths (short: 300-600 bp; long: $600-1000 \mathrm{bp}$ ) and at two different stringencies (moderate: hybridization in 5xSSC, 50\% formamide at $55^{\circ} \mathrm{C}$, wash in $0.2 \times S S C$ at $55^{\circ} \mathrm{C}$; high: hybridization in $5 \times S S C, 60 \%$ formamide at $70^{\circ} \mathrm{C}$, wash in $0.1 \times S S C$ at $72^{\circ} \mathrm{C}$ ). Under all conditions, identical results were obtained. DNp63 protein was detected with the mouse anti-p63 antibody 4A4 (Santa Cruz) as previously described (Bakkers et al., 2005). For in situ hybridisations the following riboprobes were used: col1a1 (Fisher et al., 2003; Jänicke et al., 2007), atp1b1band atp6v1a/(Jänicke etal., 2007), trpv6and pvalb8(clone IRAKp961C14226Q and IRBOp991F0754D, ImaGene, Germany; probe synthesis by PCR following the protocol on ZFIN.org). For the foxi3a, foxi3b, grh/1, grh/2a, grh/2b, grh/3, cp2, cp2-like1, Ibp-1a, prox1, NCX1b, NCC22briboprobes, RT-PCR fragments (see above) were ligated into pGEMTeasy (Promega), and plasmids were linearised and transcribed with enzymes listed in Supplementary Table 1. For single in situ hybridisations, probes were labelled with digoxygenin (Roche). In addition, for double in situ hybridisations, riboprobes for foxi3a, foxi3b, grh/1, atp1b1band atp6v1al were labelled with fluorescein (Roche).

Fluorescent double in situhybridisations were performed as described previously (Jänicke et al., 2007). Photographs were taken with a Zeiss LSM510 META confocal microscope.

\section{Bromodeoxyuridine (BrdU) labelling}

Embryos were dechorionated on petri dishes coated with $1 \%$ agarose and incubated in $2 \mathrm{mg} / \mathrm{ml} \mathrm{BrdU}+2 \%$ DMSO in embryo medium from 16 hpf (15-somites stage) to $24 \mathrm{hpf}\left(30\right.$-somites stage) at $28^{\circ} \mathrm{C}$. At $24 \mathrm{hpf}$ embryos were briefly washed in embryo medium and fixed in 4\% PFA at $4^{\circ} \mathrm{C}$ over night. For detection, in situ hybridisation was performed as before. Digoxygenin-labelled probes were developed with FastRed (Sigma). Once the desired staining intensity was achieved, embryos were washed twice for $5 \mathrm{~min}$ in PBST and incubated in $2 \mathrm{~N} \mathrm{HCl}$ for $1 \mathrm{~h}$ at $37^{\circ} \mathrm{C}$. After 5 washes with PBST for 5 min each, embryos were blocked in PBST 
supplemented with $2 \%$ sheep serum/ and $2 \mathrm{mg} / \mathrm{ml} \mathrm{BSA}$ for $1 \mathrm{~h}$ at $\mathrm{RT}$, and incubated in anti-BrdU-Alexa488 antibody (Molecular Probes) at a 1:100 dilution at $4^{\circ} \mathrm{C}$ over night. Stainings were analysed and photographs taken with a Zeiss LSM510 META confocal microscope.

\section{Morpholino and synthetic mRNA injections}

For loss-of-function analysis antisense morpholinos (MO) were purchased from GeneTools and injected into 1-2 cell stages as described previously (Nasevicius and Ekker, 2000). Sequences of foxi3-MOs and p53-MO were described previously (Jänicke et al., 2007; Plaster et al., 2006). Two grh/1-MOs were used in this work, targeting the splice donor of intron 3-4 or the ATG start codon, respectively. The sequences were: CTT TGA TGA GAG CTT CAC CTT TTG T (grh/1-splice-MO) and GTG ACA TCT CTT ATG GTC GAA CTG G (grh/1-ATG-MO). Unless stated otherwise, the ATG-MO was used. Per embryo $3 \mathrm{ng}$ of MO were injected (foxi3a, foxi3b, grh/1-splice, grh/1-ATG).

For grh/1 overexpression, the coding region of $g r h / 1$ was amplified via RT-PCR with the primers listed in Supplementary Table 1, cloned into the plasmid pCS2+ (grhl1-FL). To validate the specificity and efficacy of the grh/1-ATG-MO, the MO binding site and its flanking up- and downstream regions were amplified from cDNA with primers listed in Supplementary Table 1. The sequence was cloned into the XLT.GFP.CS2+ vector (generous gift of Jeff Miller), so that the ATG translation initiation codon of the amplified sequence was in frame and upstream of the GFP coding sequence (grhl1-GFP). pCS2-based plasmids were linearised with ECOR and capped RNA was synthesised using the T3 message machine kit (Ambion, USA). Synthetic mRNA was injected into the 1-cell stage either alone or combined with the specific ATG-MO, targeting the cloned sequence, or a control ATG-MO, targeting a different sequence. Embryos were screened for GFP at late blastula stages using a dissecting microscope. At the MO concentrations used in this work, the MO efficiently inhibited translation of GFP from the specific construct, while translation was not inhibited upon injection with an unspecific MO.

To control for the efficacy of the grh/1-splice MO, RT-PCR was performed on RNA extracted from wild-type or morphant embryos with primers binding in exon3, grhl1-ex3-for (GAC AAG AGA ACA ATA TCC CAG C) and in intron3-4, grhl1-int3/4-rev (TCA AAA ATC CTA TCA GTA AAT TT).

\section{Lineage tracing experiments}

Lineage tracing experiments were performed as previously described (Jänicke et al., 2007), transplanting single cells from bactin:mGFPtransgenic donor embryos (Cooper et al., 2005) into non-transgenic hosts. To obtain single cells, up to 10 cells were pulled from the donor embryo into the transfer needle and separated from each other by multiple up- and down movements within the pipette. Afterwards, all but one cell were released into the medium under optical control, and the remaining single cell was transferred into the host embryo. Recipients were fixed at $24 \mathrm{hpf}$, followed by fluorescent in situ hybridisation for pvalb8 as described above, by fluorescent immunolabelling of keratinocytes (primary mouse-anti-p63 antibody 4A4 (Santa Cruz) and anti-mouse-Alexa647; Molecular Probes), and by fluorescent immunolabelling of the descendants of transplanted cells (primary rabbit anti-GFP antibody and secondary anti-rabbit-Alexa488 antibody, Molecular Probes).

\section{Osmoregulation experiments}

For morphology analysis, fish were injected with MOs as described above and raised in 1x Embryo medium until the day of analysis. For osmotic stress experiments, fish were injected and raised in 1x Embryo medium until $24 \mathrm{hpf}$. They were removed from their chorions and transferred into 10x Embryo Medium for $24 \mathrm{hpf}$. At $48 \mathrm{hpf}$, the medium was replaced by distilled water. Morphology and survival rate were assessed at 72 hpf.

\section{Quantification of gene expression}

For quantification of marker expression all cells covering both sides of the trunk and the yolk sac extension of WT and grh/1 morphant embryos at $24 \mathrm{hpf}$ and $72 \mathrm{hpf}$ were counted for 5 embryos each time. For quantification of co-expression all cells covering the anterior part of the trunk and the entire yolk sac extension of WT and grh/1 morphant embryos at $24 \mathrm{hpf}$ were counted for 10 embryos each time. Analysis was performed using Excel software; statistical significance was determined using the Student's $t$ test.

\section{Database searches}

The zebrafish assembly version 7 (Zv7) was searched by BlastN and TBlastN (Altschul et al., 1997), using D-grh, D-Cp2 and the six human genes as a query. The GenBank accession numbers of the zebrafish grhl/ $\mathrm{cp} 2$ genes identified in this study as well as previously published homologues are listed in Supp-Table 2. For identification of zebrafish ncc genes, searches were performed using the human SLC12A3(NM_000339) as a query. This identified a putative ncc gene on chromosome 18 (NM 001045080). When this sequence was used in an additional search, 3 more putative nccs were identified on chromosome 22, termed here ncc22a (XM686141), ncc22b (NM001045001) and ncc22c (XM001342852). Primers were designed for all four candidates, but only $n c c 22 b$ could be amplified from 2 dpf cDNA.

\section{Phylogeny analysis}

All sequence analyses were performed using programs from the PHYLIP package (version 3.5c) (Felsenstein, 1989) available on the Mobyle portal of the Pasteur Institute, Paris (http://bioweb2.pasteur.fr/ intro-en.html). Protein sequences were aligned using ClustalW (Thompson et al., 1994). For construction of trees, regions that could not be aligned across all sequences were discarded from the alignment. For calculation of the trees, a discrete data method, parsimony, and a distance-matrix method, neighbor-joining, were compared; neighborjoining created trees with highest bootstrap values. Statistical significance was assessed by 500 bootstrap replications (Thompson et al., 1994) and percent values were included at the major branching points in the tree.

\section{Acknowledgments}

We thank Donatus Boensch and his team for excellent fish care. M.J. is a member of the Graduiertenkolleg1104 of the Faculty of Biology at the University of Freiburg. Work in the laboratory of M.H. was supported by the Max-Planck-Society, the Deutsche Forschungsgemeinschaft (SFB 829), the National Institute of Health (NIH grant 1R01-GM63904) and the European Union ( $6^{\text {th }}$ framework integrated project "Zebrafish models for human development and disease").

\section{References}

ALMEIDA, M.S. and BRAY, S.J. (2005). Regulation of post-embryonic neuroblasts by Drosophila Grainyhead. Mech Dev 122: 1282-1293.

ALTSCHUL, S.F., MADDEN, T.L., SCHAFFER, A.A., ZHANG, J., ZHANG, Z., MILLER, W. and LIPMAN, D.J. (1997). Gapped BLAST and PSI-BLAST: a new generation of protein database search programs. Nucleic Acids Res 25: 33893402.

AOTA, S., NAKAJIMA, N., SAKAMOTO, R., WATANABE, S., IBARAKI, N. and OKAZAKI, K. (2003). Pax6 autoregulation mediated by direct interaction of Pax6 protein with the head surface ectoderm-specific enhancer of the mouse Pax6 gene. Dev Bio/257: 1-13.

AUDEN, A., CADDY, J., WILANOWSKI, T., TING, S.B., CUNNINGHAM, J.M. and JANE, S.M. (2006). Spatial and temporal expression of the Grainyhead-like transcription factor family during murine development. Gene Exp Patterns 6: 964-970.

BAKKERS, J., CAMACHO-CARVAJAL, M., NOWAK, M., KRAMER, C., DANGER, B. and HAMMERSCHMIDT, M. (2005). Destabilization of DeltaNp63alpha by Nedd4-mediated ubiquitination and Ubc9-mediated sumoylation, and its implications on dorsoventral patterning of the zebrafish embryo. Cel/ Cycle 4: 790800 . 
BAKKERS, J., HILD, M., KRAMER, C., FURUTANI-SEIKI, M. and HAMMERSCHMIDT, M. (2002). Zebrafish DeltaNp63 is a direct target of Bmp signaling and encodes a transcriptional repressor blocking neural specification in the ventral ectoderm. Dev Cel/2: 617-627.

BERSET, T.A., HOIER, E.F. and HAJNAL, A. (2005). The C. elegans homolog of the mammalian tumor suppressor Dep-1/Scc1 inhibits EGFR signaling to regulate binary cell fate decisions. Genes Dev. 19: 1328-1340.

BRAY, S.J., BURKE, B., BROWN, N.B. and HIRSH, J. (1989). Embryonic expression pattern of a family of Drosophila proteins that interact with a central nervous system regulatory element. Genes Dev 5: 1672-1683.

BRAY, S.J. and KAFATOS, F.C. (1991). Developmental function of Elf-1: an essential transcription factor during embryogenesis in Drosophila. Genes Dev 5: $1672-1683$.

CENCI, C. and GOULD, A.P. (2005). Drosophila Grainyhead specifies late programmes of neural proliferation by regulating the mitotic activity and Hoxdependent apoptosis of neuroblasts. Development 132: 3835-3845.

CHALMERS, A.D., LACHANI, K., SHIN, Y., SHERWOOD, V., CHO, K.W. and PAPALOPULU, N. (2006). Grainyhead-like 3, a transcription factor identified in a microarray screen, promotes the specification of the superficial layer of the embryonic epidermis. Mech Dev 123: 702-718.

COOPER, M.S., SZETO, D.P., SOMMERS-HERIVEL, G., TOPCZEWSKI, J., SOLNICA-KREZEL, L., KANG, H.C., JOHNSON, I. and KIMELMAN, D. (2005). Visualizing morphogenesis in transgenic zebrafish embryos using BODIPY TR methyl ester dye as a vital counterstain for GFP. Dev. Dyn. 232: 359-368.

DYNLACHT, B., ATTARDI, L., ADMON, A., FREEMAN, M. and TJIAN R. (1989). Functional analysis of NTF-1, a developmentally regulated Drosophila transcription factor that binds neuronal cis-elements. Genes Dev 3: 1677-1688.

ESAKI, M., HOSHIJIMA, K., NAKAMURA, N., MUNAKATA, K., TANAKA, M., OOKATA, K., ASAKAWA, K., KAWAKAMI, K., WANG, W., WEINBERG, E.S. and HIROSE, S. (2009). Mechanism of development of ionocytes rich in vacuolar-type $\mathrm{H}(+)$-ATPase in the skin of zebrafish larvae. Dev. Biol. 329: 116129.

FELSENSTEIN, J. (1989). PHYLIP - Phyloggeny Interference Package (Version 3.2). Cladistics 5: 164-166

FINK, M., FLEKNA, G., LUDWIG, A., HEIMBUCHER, T. and CZERNY, T. (2006). Improved translation efficiency of injected mRNA during early embryonic development. Dev Dyn 235: 3370-3378.

FISHER, S., JAGADEESWARAN, P., and HALPERN, M.E. (2003). Radiographic analysis of zebrafish skeletal defects. Dev Bio/264: 64-76.

HAMMERSCHMIDT, M., PELEGRI, F., MULLINS, M.C., KANE, D.A., VAN EEDEN, F.J., GRANATO, M., BRAND, M., FURUTANI-SEIKI, M., HAFFTER, P., HEISENBERG, C.-P., JIANG, Y.J., KELSH, R.N., ODENTHAL, J., WARGA, R.M. and NÜSSLEIN-VOLHARD, C. (1996). dino and mercedes, two genes regulating dorsal development in the zebrafish embryo. Development 123: 95102.

HARLAND, R. and MISHER, L. (1988). Stability of RNA in developing Xenopus embryos and identification of a destabilizing sequence in TFIIIA messenger RNA. Development 102: 837-852.

HISLOP, N.R., CADDY, J., TING, S.B., AUDEN, A., VASUDEVAN, S., KING, S.L., LINDEMAN, G.J., VISVADER, J.E., CUNNINGHAM, J.M. and JANE, S.M. (2006). Grhl3 and Lmo4 play coordinate roles in epidermal migration. Dev Biol 321: 263-272.

HSIAO, C.D., TSAI, W.Y. and TSAI, H.J. (2002). Isolation and expression of two zebrafish homologues of parvalbumin genes related to chicken CPV3 and mammalian oncomodulin. Mech Dev119 Suppl 1: S161-S166.

HSIAO, C.D., YOU, M.S., GUH, Y.J., MA, M., JIANG, Y.J. and HWANG, P.P. (2007). A positive regulatory loop between foxi3a and foxi3b is essential for specification and differentiation of zebrafish epidermal ionocytes. PLOSONE2: e302.

HUANG, J.D., DUBNICOFF, T., LIAW, G.J., BAI, Y., VALENTINE, S.A., SHIROKAWA, J.M., LENGYEL, J.A. and COUREY, A.J. (1995). Binding sites for transcription factor NTF-1/Elf1 contribute to the ventral repression of decapentaplegic. Genes Dev 9: 3177-89.

HUANG, N. and MILLER, W.L. (2000). Cloning of factors related to HIV-inducible LBP proteins that regulate steroidogenic factor-1-independent human placental transcription of the cholesterol side-chain cleavage enzyme, P450scc. J Biol
Chem 275: 2852-2858.

HWANG, P.P. and LEE, T.H. (2007). New insights into fish ion regulation and mitochondrion-rich cells. Comp Biochem Physiol A Mol Integr Physio/148: 479 497.

JÄNICKE, M., CARNEY, T.J. and HAMMERSCHMIDT, M. (2007). Foxi3 transcription factors and Notch signaling control the formation of skin ionocytes from epidermal precursors of the zebrafish embryo. Dev Bio/307: 258-271.

JONES, K.A., LUCIW, P.A. and DUCHANGE, N. (1988). Structural arrangements of transcription control domains within the 5 '-untranslated leader regions of the HIV-1 and HIV-2 promoters. Genes Dev 2: 1101-1114.

KANG, H.C., CHAE, J.H., LEE, Y.H., PARK, M.A., SHIN, J.H., KIM, S.H., YE, S.K. CHO, Y.S., FIERING, S. and KIM, C.G. (2005). Erythroid cell-specific alphaglobin gene regulation by the $\mathrm{CP} 2$ transcription factor family. $\mathrm{Mo} / \mathrm{Ce} / / \mathrm{BiO} / 25$ : 6005-6020.

KIM, C.H., HEATH, C., BERTUCH, A. and HANSEN, U. (1987). Specific stimulation of simian virus 40 late transcription in vitro by a cellular factor binding the simian virus 40 21-base-pair repeat promoter element. Proc Nat/ Acad Sci USA 84: 6025-609.

KIMMEL, C.B., WARGA, R.M. and SCHILLING, T.F. (1990). Origin and organization of the zebrafish fate map. Development 108: 581-594.

KOBAYASHI, M., NISHIKAWA, K. and YAMAMOTO, M. (2001). Hematopoietic regulatory domain of gata1 gene is positively regulated by GATA1 protein in zebrafish embryos. Development 128: 2341-2350.

KOCHILAS, L.K., POTLURI, V., GITLER, A., BALASUBRAMANIAN, K. and CHIN, A.J. (2003). Cloning and characterization of zebrafish tbx1. Gene Expr Patterns 3: 645-651.

KUDRYAVTSEVA, E.I., SUGIHARA, T.M., WANG, N., LASSO, R.J., GUDNASON, J.F., LIPKIN, S.M. and ANDERSEN, B. (2003). Identification and characterization of Grainyhead-like epithelial transactivator (GET-1), a novel mammalian Grainyhead-like factor. Dev Dyn 226: 604-617.

KURTH, I., HENTSCHE, M., HENTSCHE, S., BORGMEYER, U., GAL, A. and HÜBNER, C. (2006). The forkhead transcription factor Foxi1 directly activates the AE4 promoter. Biochem J393: 277-283.

LE BORGNE, R. and SCHWEISGUTH F. (2003). Unequal segregation of Neutralized biases Notch activation during asymmetric cell division. Dev Cel/5: 139148.

LE GUELLEC, D., MORVAN-DUBOIS, G. and SIRE, J.Y. (2004). Skin development in bony fish with particular emphasis on collagen deposition in the dermis of the zebrafish (Danio rerio). Int J Dev Bio/48: 217-231.

LEE, H. and KIMELMAN, D. (2002). A dominant-negative form of $\mathrm{p} 63$ is required for epidermal proliferation in zebrafish. Dev Cel/2: 607-616.

LIAO, B.K., DENG, A.N., CHEN, S.C., CHOU, M.Y. and HWANG, P.P. (2007). Expression and water calcium dependence of calcium transporter isoforms in zebrafish gill mitochondrion-rich cells. BMC Genomics 8: 354.

LIM, L. C., SWENDEMAN, S. L. and SHEFFERY, M. (1992). Molecular cloning of the alpha-globin transcription factor CP2. Mol Cel/ Bio/12: 828-835.

LIN, L.Y., HORNG, J.L., KUNKEL, J.G. and HWANG, P.P. (2006). Proton pump-rich cell secretes acid in skin of zebrafish larvae. Am J Physiol Cell Physiol290: C371-C378.

MANNING, L. and DOE, C.Q. (1999). Prospero distinguishes sibling cell fate without asymmetric localization in the Drosophila adult external sense organ lineage. Development 126: 2063-2071.

MAURANGE, C., CHENG, L. and GOULD, A. P. (2008). Temporal transcription factors and their targets schedule the end of neural proliferation in Drosophila. Cel/133: 891-902.

MCCLINTOCK, J.M., KHEIRBEK, M.A. and PRINCE, V.E. (2002). Knockdown of duplicated zebrafish hoxb1 genes reveals distinct roles in hindbrain patterning and a novel mechanism of duplicate gene retention. Development 129: 23392354.

MEYER, A. and SCHARTL, M. (1999). Gene and genome duplications in vertebrates: the one-to-four (-to-eight in fish) rule and the evolution of novel gene functions. Curr Opin Cell Bio/11: 699-704.

MURATA, T., NITTA, M. and YASUDA, K. (1998). Transcription factor CP2 is essential for lens-specific expression of the chicken alphaA-crystallin gene. Genes Cells 3: 443-457. 
NASEVICIUS, A. and EKKER, S.C. (2000). Effective targeted gene 'knockdown' in zebrafish. Nat Genet 26: 216-220.

NICA, G., HERZOG, W., SONNTAG, C. and HAMMERSCHMIDT, M. (2004). Zebrafish pit1 mutants lack three pituitary cell types and develop severe dwarfism. Mol Endocrinol18: 1196-1209.

NÜSSLEIN-VOLHARD, C., WIESCHAUS, E. and KLUDING, H. (1984). Mutations affecting the pattern of the larval cuticle in Drosophila melanogaster. 1. Zygotic loci on the second chromosome. Wilhelm Roux's Arch Dev Bio/193: 267-282.

PAN, T.C., LIAO, B.K., HUANG, C.J., LIN, L.Y. and HWANG, P.P. (2005). Epithelial $\mathrm{Ca}(2+)$ channel expression and $\mathrm{Ca}(2+)$ uptake in developing zebrafish. $\mathrm{Am} J$ Physiol Regul Integr Comp Physio/289: R1202-R1211.

PAULS, T.L., COX, J.A. and BERCHTOLD, M.W. (1996). The Ca2+(-)binding proteins parvalbumin and oncomodulin and their genes: new structural and functional findings. Biochim Biophys Acta 1306: 39-54.

PLASTER, N., SONNTAG, C., BUSSE, C.E. and HAMMERSCHMIDT, M. (2006). p53 deficiency rescues apoptosis and differentiation of multiple cell types in zebrafish flathead mutants deficient for DNA polymerase delta1. Cell Death Differ 13: 223-235.

POSTLETHWAIT, J.H., YAN, Y.L., GATES, M.A., HORNE, S., AMORES, A., BROWNLIE, A., DONOVAN, A., EGAN, E.S., FORCE, A., GONG, Z., GOUTEL, C., FRITZ, A., KELSH, R., KNAPIK, E., LIAO, E., PAW, B., RANSOM, D., SINGER, A., THOMSON, M., ABDULJABBAR, T.S., YELICK, P., BEIER, D., JOLY, J.S., LARHAMMAR, D., ROSA, F., WESTERFIELD, M., ZON, L.I., JOHNSON, S.L. and TALBOT, W.S. (1998). Vertebrate genome evolution and the zebrafish gene map. Nat Genet 18: 345-349.

RAMAMURTHY, L., BARBOUR, V., TUCKFIELD, A., CLOUSTON, D.R., TOPHAM, D., CUNNINGHAM, J.M. and JANE, S.M. (2001). Targeted disruption of the CP2 gene, a member of the NTF family of transcription factors. J Bio/Chem276: 7836-7842.

ROBU, M.E., LARSON, J.D., NASEVICIUS, A., BEIRAGHI, S., BRENNER, C., FARBER, S.A. and EKKER, S. C. (2007). p53 activation by knockdown technologies. PLOS Genet 3: e78.

RODDA, S., SHARMA, S., SCHERER, M., CHAPMAN, G. and RATHJEN, P. (2001). CRTR-1, a developmentally regulated transcriptional repressor related to the CP2 family of transcription factors. J Biol Chem 276: 3324-3332.

SAGERSTROM, C.G., GAMMILL, L.S., VEALE, R. and SIVE, H. (2005). Specification of the enveloping layer and lack of autoneuralization in zebrafish embryonic explants. Dev Dyn 232: 85-97.

SHIH, L.J., LU, Y.F., CHEN, Y.H., LIN, C.C., CHEN, J.A. AND HWANG, S.P. (2007). Characterization of the agr2 gene, a homologue of $X$. laevis anterior gradient 2, from the zebrafish, Danio rerio. Gene Expr Patterns 7: 452-460.

SHIN, J., POLING, J., PARK, H.C. and APPEL, B. (2007). Notch signaling regulates neural precursor allocation and binary neuronal call fate decisions in zebrafish. Development 134: 1911-1920.

SLANCHEV K., CARNEY T.J., STEMMLER M.P., KOSCHORZ B., AMSTERDAM A., SCHWARZ H. and HAMMERSCHMIDT M. (2009). The epithelial cell adhesion molecule EpCAM is required for epithelial morphogenesis and integrity during zebrafish epiboly and skin development. PLOS Genet5: e1000563

SOLOMON, K.S., LOGSDON, J.M., JR. and FRITZ, A. (2003). Expression and phylogenetic analyses of three zebrafish Foxl class genes. Dev Dyn228: 301307.

SONAWANE, M., CARPIO, Y., GEISLER, R., SCHWARZ, H., MAISCHEIN, H.M. and NÜSSLEIN-VOLHARD, C. (2005). Zebrafish penner/lethal giant larvae 2 functions in hemidesmosome formation, maintenance of cellular morphology and growth regulation in the developing basal epidermis. Development 132: 3255-3265

SUEYOSHI, T., KOBAYASHI, R., NISHIO, K., AIDA, K., MOORE, R., WADA, T., HANDA, H. and NEGISHI, M. (1995). A nuclear factor (NF2d9) that binds to the male-specific P450 (Cyp 2d-9) gene in mouse liver. Mol Cel/ Bio/15: 4158-4166.

TAO, J., KULIYEV, E., WANG, X., LI, X., WILANOWSKI, T., JANE, S.M., MEAD, P.E. and CUNNINGHAM, J.M. (2005). BMP4-dependent expression of Xenopus Grainyhead-like 1 is essential for epidermal differentiation. Development 132: $1021-1034$.
THISSE, B., PFLUMIO, S., FÜRTHAUER, M., LOPPIN, B., HEYER, V., DEGRAVE, A., WOEHL, R., LUX, A., STEFFAN, T., CHARBONNIER, X.Q. and THISSE, C. (2001). Expression of the zebrafish genome during embryogenesis (NIH R01 RR15402). ZFIN Direct Data Submission (http://zfin.org).

THOMPSON, J.D., HIGGINS, D.G. and GIBSON, T.J. (1994). CLUSTAL W: improving the sensitivity of progressive multiple sequence alignment through sequence weighting, position-specific gap penalties and weight matrix choice. Nucleic Acids Res 22: 4673-4680.

TING, S.B., WILANOWSKI, T., CERRUTI, L., ZHAO, L.L., CUNNINGHAM, J.M and JANE, S.M. (2003). The identification and characterization of human Sisterof-Mammalian Grainyhead (SOM) expands the grainyhead-like family of developmental transcription factors. Biochem J370: 953-962.

TING, S.B., CADDY, J., HISLOP, N., WILANOWSKI, T., AUDEN, A., ZHAO, L.L., ELLIS, S., KAUR, P., UCHIDA, Y., HOLLERAN, W.M., ELIAS, P.M., CUNNINGHAM, J.M. and JANE, S.M. (2005). A homolog of Drosophila grainyhead is essentail for epidermal integrity in mouse. Science 308: 411-413.

UV, A.E., HARRISON, E.J. and BRAY, S.J. (1997). Tissue-specific splicing and functions of the Drosophila transcription factor Grainyhead. Mol Cel/ Bio/17: 6727-6735.

VAN LAER, L., VAN EYKEN, E., FRANSEN, E., HUYGHE, J.R., TOPSAKAL, V., HENDRICKX, J.J., HANNULA, S., MAKI-TORKKO, E., JENSEN, M., DEMEESTER, K., ET AL. (2008). The grainyhead like 2 gene (GRHL2), alias TFCP2L3, is associated with age-related hearing impairment. Hum Mol Genet 17: 159-169.

VARSAMOS, S., NEBEL, C. and CHARMANTIER, G. (2005). Ontogeny of osmoregulation in postembryonic fish: a review. Comp Biochem Physiol A Mol Integr Physiol141: 401-429.

VENKATESAN, K., MCMANUS, H.R., MELLO, C.C., SMITH, T.F. and HANSEN, U (2003). Functional conservation between members of an ancient duplicated transcription factor family, LSF/Grainyhead. Nucleic Acids Res31: 4304-4316.

WHITEAR, M. (1986). The skin of fishes including cyclostomes: Epidermis. In Biology of the Integument 2 Vertebrates (Eds. J. Bereiter-Han, A.G. Matoltsy and K.S. Richards), pp. 8-38; Springer-Verlag, Berlin, Heidelberg, New York, Tokyo.

WILANOWSKI, T., TUCKFIELD, A., CERRUTI, L., O'CONNELL, S., SAINT, R., PAREKH, V., TAO, J., CUNNINGHAM, J.M. and JANE, S.M. (2002). A highly conserved novel family of mammalian developmental transcription factors related to Drosophila grainyhead. Mech Dev 114: 37-50.

WILANOWSKI, T., CUDDY, J., TING, S.B., HISLOP, N.R., CERRUIT, L., AUDEN, A., ZHAO, L.L., ASQUITH S., ELLIS, S., SINCLAIR, R., CUNNINGHAM, J.M. and JANE, S.M. (2008). Perturbed desmosomal cadherin expression in grainy head-like a-null mice. EMBO J27: 886-897.

WU, F.K., GARCIA, J.A., HARRICH, D. and GAYNOR, R.B. (1988). Purification of the human immunodeficiency virus type 1 enhancer and TAR binding proteins EBP-1 and UBP-1. EMBO J7: 2117-2130.

XU, Y., WANG, S., ZHANG, J., ZHAO, A., STANGER, B.Z. and GU, G. (2006). The fringe molecules induce endocrine differentiation in embryonic endoderm by activating cMyt1/cMyt3. Dev Bio/297: 340-349.

YAMAGUCHI, Y., YONEMURA, S. and TAKADA, S. (2006). Grainyhead-related transcription factor is required for duct maturation in the salivary gland and the kidney of the mouse. Development 133: 4737-4748.

YU, Z., BHANDARI, A., MANNIK, J., PHAM, T., XU, X. and ANDERSEN, B. (2008). Grainyhead-like factor Get1/Grhl3 regulates formation of the epidermal leading edge during eyelid closure. Dev Bio/319: 56-67.

YU, Z., LIN, K.K., BHANDARI, A., SPENCER, J.A., XU, X., WANG, N., LU, Z., GILL, G.N., ROOP, D.R., WERTZ, P. and ANDERSEN, B. (2006). The Grainyheadlike epithelial transactivator Get- $1 / \mathrm{Grh} / 3$ regulates epidermal terminal differentiation and interacts functionally with LMO4. Dev Bio/299: 122-136.

ZACCHIA, M. and CAPASSO, G. (2008). Parvalbumin: a key protein in early distal tubule $\mathrm{NaCl}$ reabsorption. Nephrol Dial Transplant 23: 1109-1111.

ZHAO, R., WATT, A., LI, J., LUEBKE-WHEELER, J., MORRISEY, E.E. and DUNCAN, S.A. (2005). GATA6 is essential for embryonic development of the liver but dispensable for early heart formation. Mol Cel/ Bio/25: 2622-2631. 


\section{Further Related Reading, published previously in the Int. J. Dev. Biol.}

See Special Issue Pattern Formation edited by Michael K. Richardson and Cheng-Ming Chuong at:

http://www.ijdb.ehu.es/web/contents.php?vol=53\&issue=5-6

Human conjunctival epithelial precursor cells and their progeny in 3D organotypic culture

Alfredo Rosellini, Sandra Papini, Claudio Giannarini, Marco Nardi and Roberto P. Revoltella Int. J. Dev. Biol. (2007) 51: 739-743

Notch in vertebrates - molecular aspects of the signal

Ken-Ichi Katsube and Kei Sakamoto

Int. J. Dev. Biol. (2005) 49: 369-374

Scale development in fish: a review, with description of sonic hedgehog (shh) expression in the zebrafish (Danio rerio)

Jean-Yves Sire and Marie-Andrée Akimenko

Int. J. Dev. Biol. (2004) 48: 233-247

Skin development in bony fish with particular emphasis on collagen deposition in the dermis of the zebrafish (Danio rerio)

Dominique Le Guellec, Ghislaine Morvan-Dubois and Jean-Yves Sire

Int. J. Dev. Biol. (2004) 48: 217-231

Derivation of keratinocyte progenitor cells and skin formation from embryonic stem cells

Daniel Aberdam

Int. J. Dev. Biol. (2004) 48: 203-206

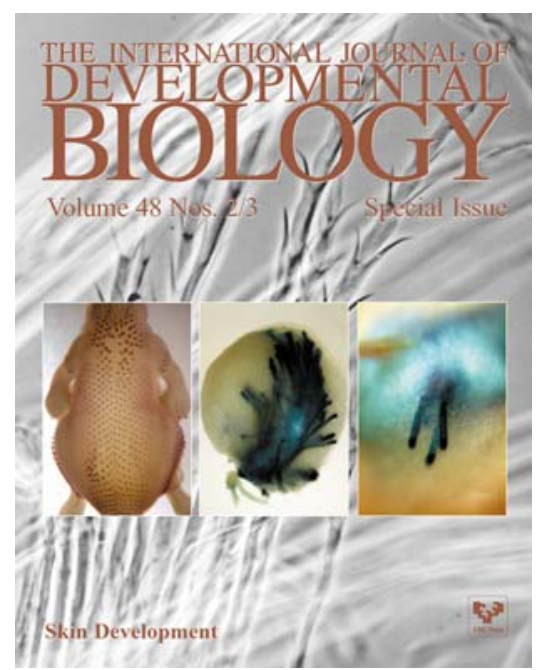

5 yr ISI Impact Factor $(2008)=3.271$

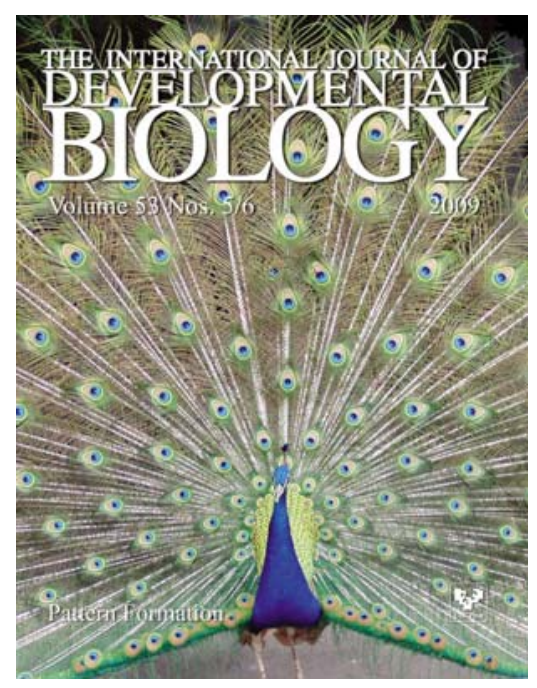

